\title{
Insights into Fast Amide Couplings in Aqueous Nanomicelles
}

Sudripet Sharma, ${ }^{\dagger}$ Gaganpreet Kaur, ${ }^{\dagger}$ Sachin Handa ${ }^{\dagger, *}$

${ }^{\dagger}$ Department of Chemistry, University of Louisville, Louisville, Kentucky 40292, United States

*Email: sachin.handa@louisville.edu

\section{Supporting Information}

\section{Contents}

1. General experimental details

2. HRTEM analysis

3. Kinetic studies - effect of base

4. Kinetic studies - reaction in aqueous PS-750-M versus neat $\mathrm{H}_{2} \mathrm{O}$

5. Synthesis of intermediate 6 for efaproxiral

6. Synthesis of intermediate 8 for ACT-442206

7. Multigram synthesis of $\mathbf{3}$

8. Multigram synthesis of $\mathbf{1 1}$

9. Epimerization studies

10. NMR experiments

11. Control experiment - reaction without base

12. References
Page

S1

S2

S3-S4

S5-S6

S7-S9

$\mathrm{S} 10-\mathrm{S} 13$

S14-S16

S17-S19

S20-S23

S24-S27

S28

S29 


\section{GENERAL EXPERIMENTAL DETAILS}

TLC plates (UV 254 indicator, glass backed, thickness $200 \mathrm{~mm}$ ) were purchased from SigmaAldrich and silica gel (standard grade, 230 - 400 mesh) was purchased from Silicycle. Pyridine was purchased from Fisher Scientific and used as such without further purification. Pure NMR solvents were purchased from Cambridge Isotopes Laboratories. Carboxylic acids and amines were purchased from Sigma-Aldrich, Combi-Block, and Oakwood chemicals. EDC $\bullet \mathrm{HCl}$ and other coupling reagents were purchased from Oakwood chemicals. Coupling reactions were performed in $4 \mathrm{~mL}$ close-cap microwave vials and $250 \mathrm{~mL}$ round bottom flasks. Microwave vials were purchased from VWR International and Biotage. Reaction vials were also recycled and reused. Bulk aqueous solution of surfactant PS-750-M $\mathrm{M}^{1}$ was prepared in HPLC grade water and thoroughly purged with argon before use. Unless otherwise mentioned, all NMR spectra were recorded at 25 ${ }^{\circ} \mathrm{C}$ on Varian Unity INOVA (400 and $500 \mathrm{MHz}$ ) spectrometers. Reported chemical shifts are referenced to residual solvent peaks. ${ }^{2}$ 


\section{HRTEM ANALYSIS}

Analyte was prepared by dissolving $40 \mathrm{mg} \mathrm{EDC} \cdot \mathrm{HCl}$ in $0.5 \mathrm{~mL} 3$ wt \% aq. PS-750-M. The resulting mixture was analyzed by high-resolution transmission electron microscopy (HRTEM). A $3 \mu \mathrm{L}$ aliquot of the sample was applied to a glow-discharged ultra-thin carbon TEM grid and stained by $2 \%$ uranyl formate. The grid was then transferred to a FEI TF30 field emission gun transmission electron microscope (Hillsboro, Oregon). The image was recorded at a nominal magnification of 4700x using a K2 Summit direct electron detector camera (Gatan, INC., Pleasanton, CA).

The average particle size was calculated from these images and histogram was obtained for the size distribution. The size of the particles ranges from $17 \mathrm{~nm}$ to $170 \mathrm{~nm}$. The average size is 92 nm.
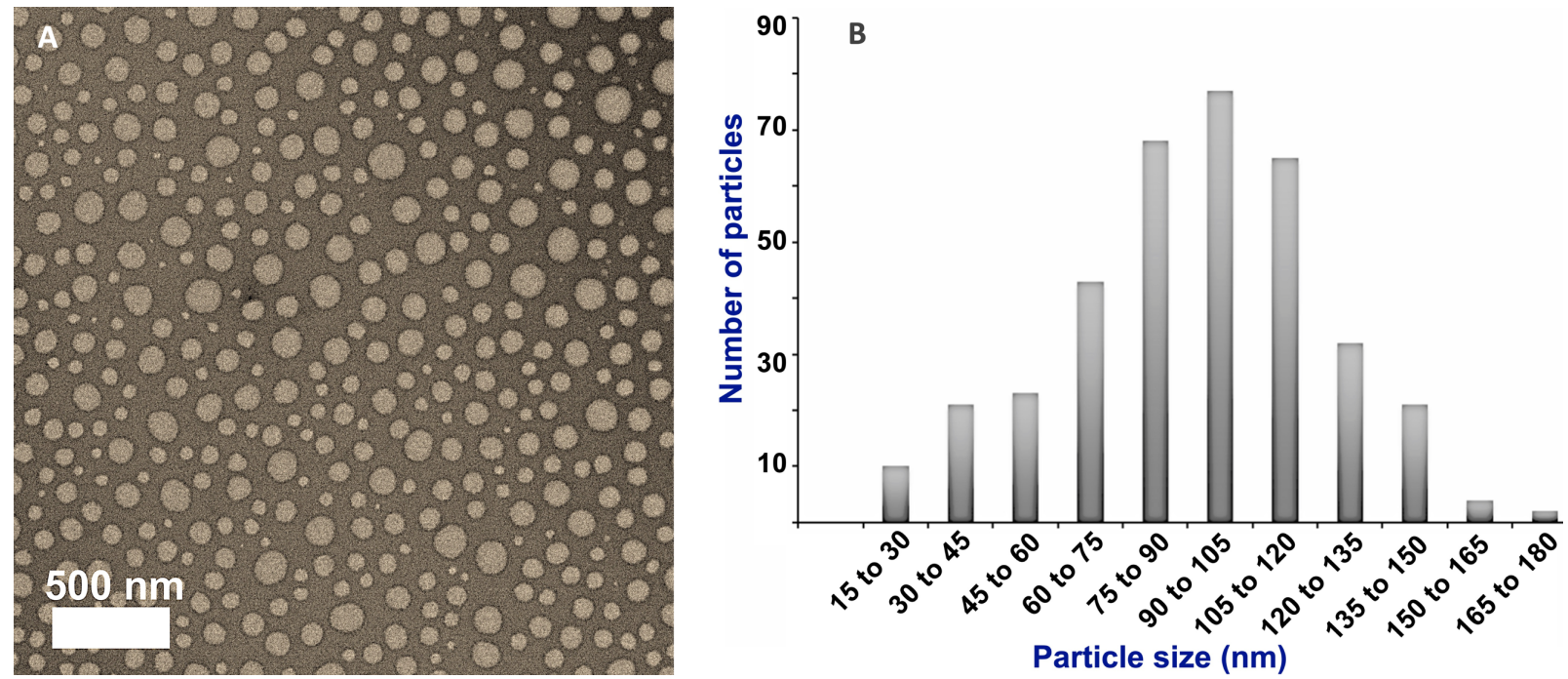

Figure S1. (A) HRTEM image of $\mathrm{EDC} \cdot \mathrm{HCl}$ in 3 wt \% aqueous PS-750-M; (B) particle size distribution. 


\section{KINETIC PROFILE WITH RESPECT TO THE NATURE OF BASE}
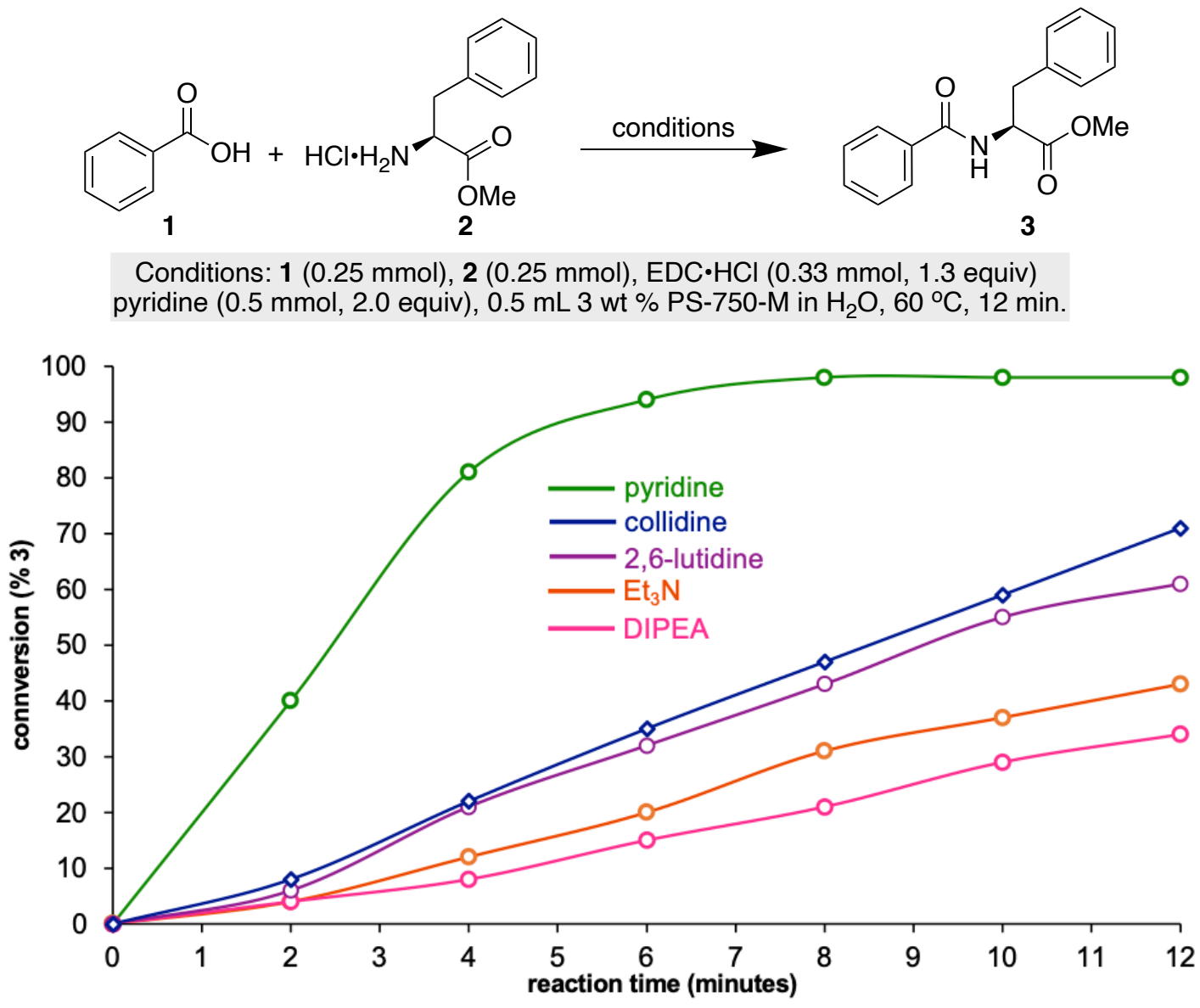

Figure S2. Kinetic profile with respect to the nature of base.

General procedure for kinetic studies. Benzoic acid (1) (30.5 mg, $0.25 \mathrm{mmol})$, methyl $L$ phenylalaninate hydrochloride (2) $(54 \mathrm{mg}, 0.25 \mathrm{mmol})$, and $\mathrm{EDC} \cdot \mathrm{HCl}(73 \mathrm{mg}, 0.325 \mathrm{mmol}, 1.3$ equiv.) were taken in an oven-dried $4 \mathrm{~mL}$ reaction vial equipped with a PTFE-coated magnetic stir bar. Reaction flask was closed with a rubber septum and $0.5 \mathrm{~mL} 3 \mathrm{wt} \%$ aqueous PS-750-M was added to the reaction mixture. Septum was wrapped with PTFE tape and a parafilm. Base $(0.5$ mmol, 2 equiv.) was subsequently added to the reaction mixture and mixture was allowed to stir at $60{ }^{\circ} \mathrm{C}$. The reaction progress was analyzed using GC-MS by using 1,1,2,2-tetrachloroethane $(0.25 \mathrm{mmol})$ as an internal standard as described below:

(a) Reaction kinetics using pyridine as a base. A set of six reactions were setup according to general procedure (described above) using pyridine ( $0.5 \mathrm{mmol}, 2$ equiv.) as a base. After 2 minutes, one reaction vial was immediately cooled to $0{ }^{\circ} \mathrm{C}$ and $1 \mathrm{~mL}$ EtOAc along with 1,1,2,2- 
tetrachloroethane $(0.25 \mathrm{mmol})$ as an internal standard was added to it. A reaction mixture was stirred for 2 minutes at $0{ }^{\circ} \mathrm{C}$ and the reaction progress and formation of $\mathbf{3}$ was analyzed using GCMS. A same analysis procedure was applied to remaining five reactions at 4, 6, 8, 10, and 12 minutes time interval, respectively.

(b) Reaction kinetics using collidine as a base. A set of six reactions were setup according to general procedure (described above) using collidine ( $0.5 \mathrm{mmol}, 2$ equiv.) as a base. After 2 minutes, one reaction vial was immediately cooled to $0{ }^{\circ} \mathrm{C}$ and $1 \mathrm{~mL}$ EtOAc along with 1,1,2,2tetrachloroethane $(0.25 \mathrm{mmol})$ as an internal standard was added to it. A reaction mixture was stirred for 2 minutes at $0{ }^{\circ} \mathrm{C}$ and the reaction progress and formation of $\mathbf{3}$ was analyzed using GCMS. A same analysis procedure was applied to remaining five reactions at 4, 6, 8, 10, and 12 minutes time interval, respectively.

(c) Reaction kinetics using 2,6-lutidine as a base. Six set of reactions were setup according to general procedure (described above) using 2,6-lutidine ( $0.5 \mathrm{mmol}, 2$ equiv.) as a base. After 2 minutes, one reaction vial was immediately cooled to $0{ }^{\circ} \mathrm{C}$ and $1 \mathrm{~mL}$ EtOAc along with 1,1,2,2tetrachloroethane $(0.25 \mathrm{mmol})$ as an internal standard was added to it. A reaction mixture was stirred for 2 minutes at $0{ }^{\circ} \mathrm{C}$ and the reaction progress and formation of $\mathbf{3}$ was analyzed using GCMS. The same procedure was repeated for other set of reactions in every 2 minutes time interval. (d) Reaction kinetics using triethylamine as a base. A set of six reactions were setup according to general procedure (described above) using $\mathrm{Et}_{3} \mathrm{~N}$ ( $0.5 \mathrm{mmol}, 2$ equiv.) as a base. After 2 minutes, one reaction vial was immediately cooled to $0{ }^{\circ} \mathrm{C}$ and $1 \mathrm{~mL}$ EtOAc along with 1,1,2,2tetrachloroethane $(0.25 \mathrm{mmol})$ as an internal standard was added to it. A reaction mixture was stirred for 2 minutes at $0{ }^{\circ} \mathrm{C}$ and the reaction progress and formation of $\mathbf{3}$ was analyzed using GCMS. A same analysis procedure was applied to remaining five reactions at 4, 6, 8, 10, and 12 minutes time interval, respectively.

(e) Reaction kinetics using diisopropylethylamine as a base. A set of six reactions were setup according to general procedure (described above) using $i$ - $\operatorname{Pr}_{2} \mathrm{NEt}(0.5 \mathrm{mmol}, 2$ equiv.) as a base. After 2 minutes, one reaction vial was immediately cooled to $0{ }^{\circ} \mathrm{C}$ and $1 \mathrm{~mL}$ EtOAc along with 1,1,2,2-tetrachloroethane $(0.25 \mathrm{mmol})$ as an internal standard was added to it. A reaction mixture was stirred for 2 minutes at $0{ }^{\circ} \mathrm{C}$ and the reaction progress and formation of $\mathbf{3}$ was analyzed using GC-MS. A same analysis procedure was applied to remaining five reactions at 4, 6, 8, 10, and 12 minutes time interval, respectively. 


\section{KINETIC STUDIES- REACTION IN AQUEOUS PS-750-M VERSUS NEAT H2O}

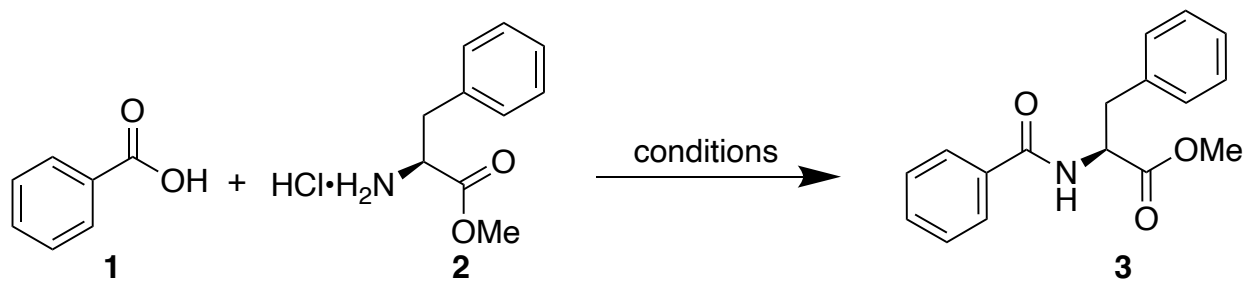

Conditions: $1(0.25 \mathrm{mmol}), 2(0.25 \mathrm{mmol}), \mathrm{EDC} \cdot \mathrm{HCl}(0.33 \mathrm{mmol}, 1.3$ equiv) pyridine ( $0.5 \mathrm{mmol}$, 2.0 equiv), $0.5 \mathrm{~mL} 3 \mathrm{wt} \%$ PS-750- $\mathrm{M}$ in $\mathrm{H}_{2} \mathrm{O}, 60^{\circ} \mathrm{C}, 12 \mathrm{~min}$.

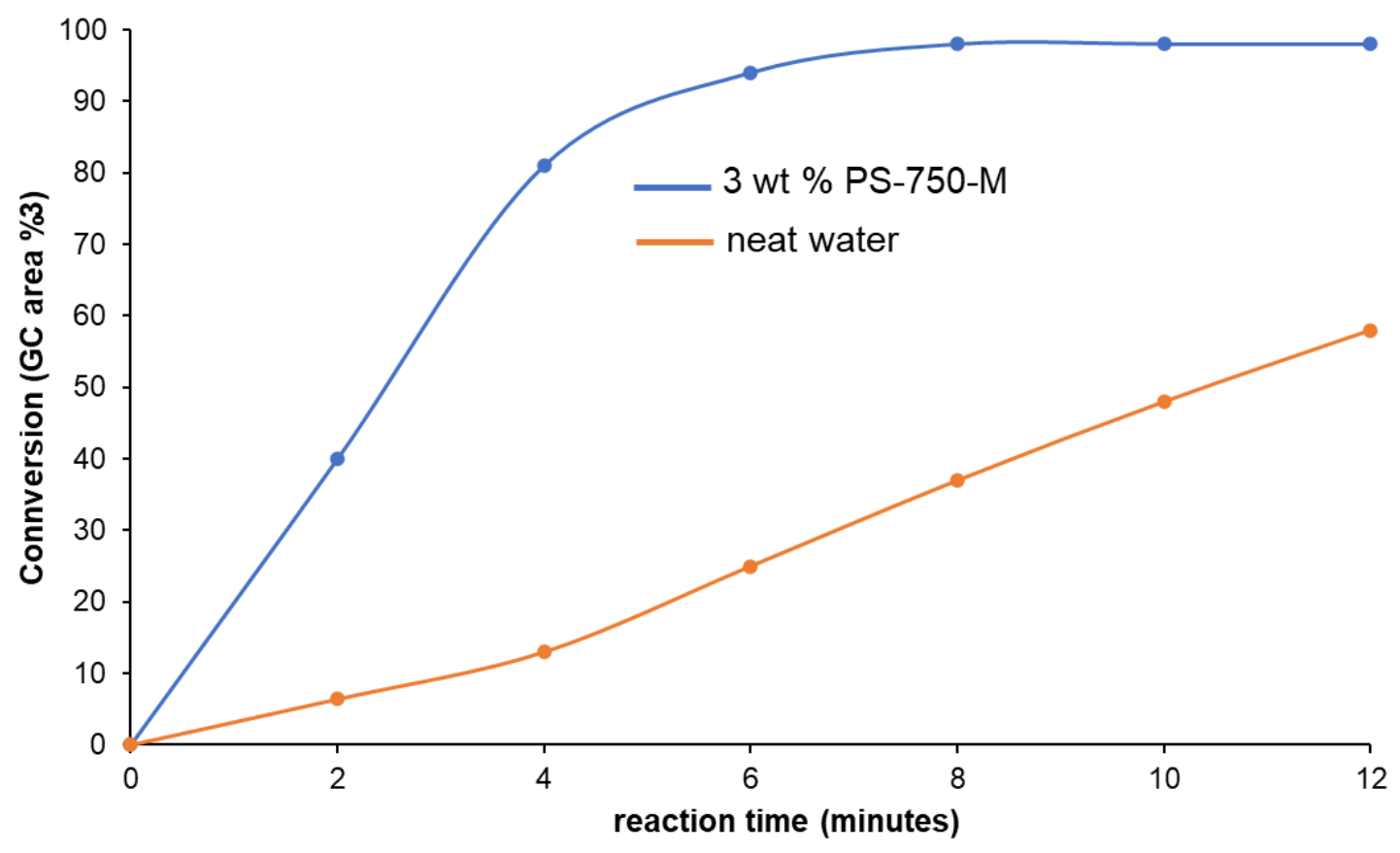

Figure S3. Reaction kinetics in 3 wt \% PS-750-M versus neat $\mathrm{H}_{2} \mathrm{O}$.

General procedure. Benzoic acid (1) (30.5 mg, $0.25 \mathrm{mmol}$ ), methyl $L$-phenylalaninate hydrochloride (2) (54 mg, $0.25 \mathrm{mmol}$ ), and $\mathrm{EDC} \cdot \mathrm{HCl}$ (73 mg, $0.325 \mathrm{mmol}, 1.3$ equiv.) were taken in an oven-dried $4 \mathrm{~mL}$ reaction vial equipped with a PTFE-coated magnetic stir bar. Reaction flask was closed with a rubber septum and $0.5 \mathrm{~mL}$ solvent (3 wt \% aqueous PS-750-M or de-ionized water) was added to the reaction mixture. Septum was wrapped with PTFE tape and a parafilm. Pyridine ( $0.5 \mathrm{mmol}, 2$ equiv.) was subsequently added to the reaction mixture and mixture was allowed to stir at $60{ }^{\circ} \mathrm{C}$. The reaction progress was analyzed using GC-MS by adding 1,1,2,2tetrachloroethane $(0.25 \mathrm{mmol})$ as an internal standard. The details of kinetic experiments are provided below: 
(a) Reaction kinetics using 3 wt \% aqueous PS-750-M as reaction medium. A set of six reactions were setup according to general procedure (described above) using $3 \mathrm{wt} \% \mathrm{PS}-750-\mathrm{M}$ as reaction medium. After 2 minutes, one reaction vial was immediately cooled to $0{ }^{\circ} \mathrm{C}$ and $1 \mathrm{~mL}$ EtOAc along with 1,1,2,2-tetrachloroethane $(0.25 \mathrm{mmol})$ as an internal standard was added to it. A reaction mixture was stirred for 2 minutes at $0{ }^{\circ} \mathrm{C}$ and the reaction progress and formation of 3 was analyzed using GC-MS. The same analysis procedure was applied to remaining five reactions at 4, 6, 8, 10, and 12 minutes time interval, respectively.

(b) Reaction kinetics using de-ionized $\mathrm{H}_{2} \mathrm{O}$ as reaction medium. A set of six reactions were setup according to general procedure (described above) using de-ionized $\mathrm{H}_{2} \mathrm{O}$ as reaction medium. After 2 minutes, one reaction vial was immediately cooled to $0{ }^{\circ} \mathrm{C}$ and $1 \mathrm{~mL}$ EtOAc along with 1,1,2,2-tetrachloroethane $(0.25 \mathrm{mmol})$ as an internal standard was added to it. A reaction mixture was stirred for 2 minutes at $0{ }^{\circ} \mathrm{C}$ and the reaction progress and formation of $\mathbf{3}$ was analyzed using GC-MS. A same analysis procedure was applied to remaining five reactions at 4, 6, 8, 10, and 12 minutes time interval, respectively. 


\section{MULTI-GRAM SCALE SYNTHESIS OF INTERMEDIATE 6 FOR EFAPROXIRAL}

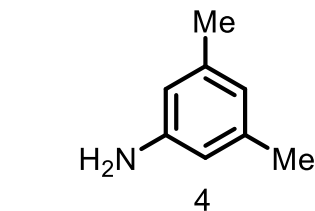

$8 \mathrm{~g}(65.7 \mathrm{mmol}, 1.0$ equiv.)

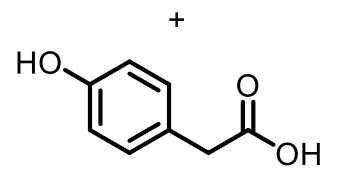

5

$10 \mathrm{~g}(65.7 \mathrm{mmol}, 1.0$ equiv $)$

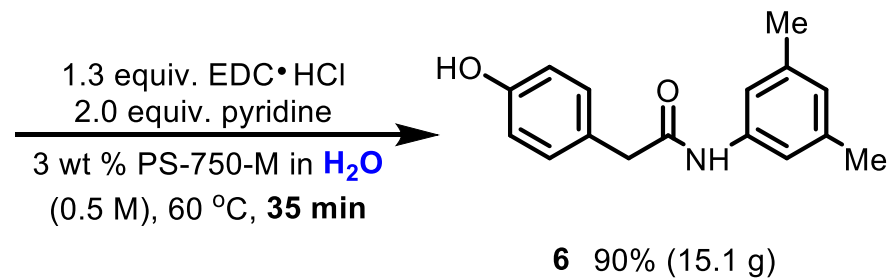

$690 \%(15.1 \mathrm{~g})$

Scheme S1. Multi-gram scale synthesis of intermediate 6 for efaproxiral.

4-Hydroxyphenylacetic acid (5) (10 g, 65.7 mmol, 1 equiv.), 3,5-dimethylaniline (4) (7.96 g, 65.7 mmol, 1 equiv.) and $\mathrm{EDC} \cdot \mathrm{HCl}(16.4 \mathrm{~g}, 85.4 \mathrm{mmol}, 1.3$ equiv.) were taken in an oven-dried 500 $\mathrm{mL}$ round-bottom flask equipped with a PTFE-coated magnetic stir bar. Reaction flask was closed with a rubber septum and $130 \mathrm{~mL} 3 \mathrm{wt} \%$ aqueous PS-750-M was added to the reaction mixture. Septum was wrapped with PTFE tape and a parafilm. Pyridine (10.6 mL, 131.4 mmol, 2 equiv.) was subsequently added to the reaction mixture and mixture was stirred at $60{ }^{\circ} \mathrm{C}$ on pre-heated oil bath for 35 minutes. A solid formation was observed in the reaction mixture. After reaction completion as monitored by TLC (ethyl acetate/hexane, 2:3), stirring was stopped and reaction mixture was cooled to rt. Solid was filtered through a Whatman filter paper and washed with deionized water $(3 \times 30 \mathrm{~mL})$. Collected solid was dried under reduced pressure to obtain $N-(3,5-$ dimethylphenyl)-2-(4-hydroxyphenyl)acetamide (6) as final product. ${ }^{2}$ Off-white solid, yield 15.1 g (90\%), R $\mathrm{R}_{f} 0.4$ (2:3, hexanes/ethyl acetate). ${ }^{\mathbf{1} H}$ NMR (400 MHz, DMSO-d $)$ : $\delta 9.89$ (s, $\left.1 \mathrm{H}\right), 9.24$ (s, 1H), $7.20(\mathrm{~s}, 2 \mathrm{H}), 7.10(\mathrm{~d}, J=7.9 \mathrm{~Hz}, 2 \mathrm{H}), 6.75-6.64(\mathrm{~m}, 3 \mathrm{H}), 3.45(\mathrm{~s}, 2 \mathrm{H}), 2.21(\mathrm{~s}, 6 \mathrm{H}) .{ }^{13} \mathrm{C}$ NMR (100 MHz, DMSO-d $) \delta$ 169.5, 156, 139.1, 137.6, 129.9, 126.2, 124.6, 116.8, 115, 42.6, 21.1. ${ }^{3}$ Product purity (98.07\%) was determined by HPLC with CHIRALCEL $^{\circledR}$ AD-H column (hexane $/ i-\mathrm{PrOH}=90 / 10$ ), flow rate $=1 \mathrm{~mL} / \mathrm{min}, 25^{\circ} \mathrm{C}$. Process mass intensity $(\mathrm{PMI})=17.56$ (calculated according to the reported procedure) ${ }^{4}$. 

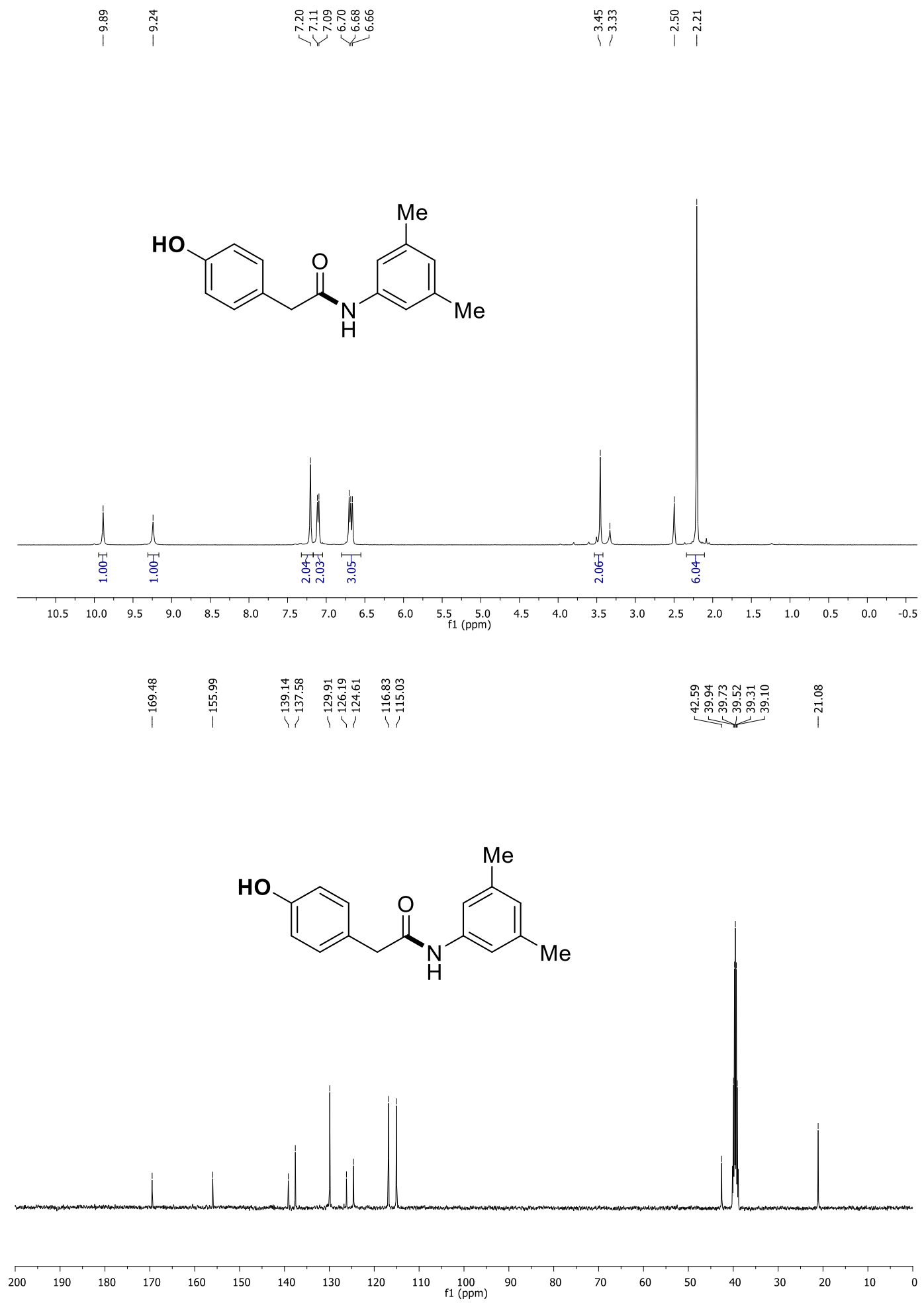

Figure S4. ${ }^{1} \mathrm{H}$ and ${ }^{13} \mathrm{C}$ NMR of 6 . 


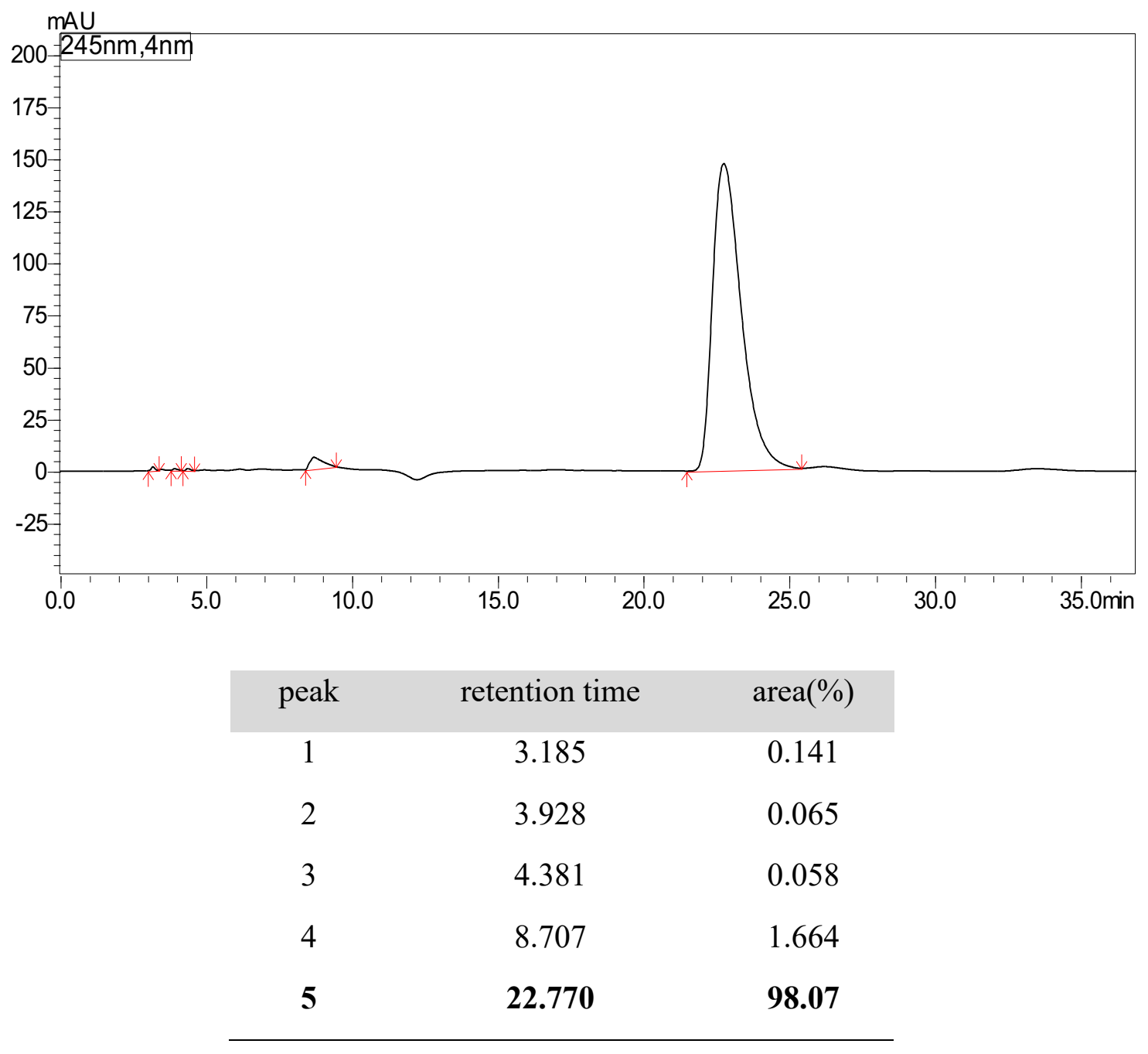

Figure S5. Purity analysis of 6 by HPLC. 


\section{MULTI-GRAM SYNTHESIS OF INTERMEDIATE 8 FOR ACT-462206}

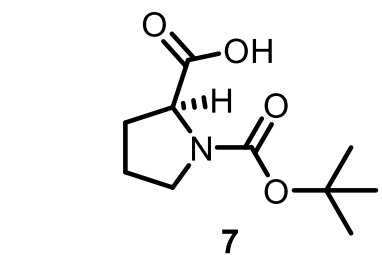

$10 \mathrm{~g}(46.5 \mathrm{mmol}, 1$ equiv.)

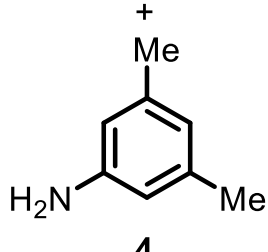

4
1.3 equiv. EDC• $\mathrm{HCl}$ 2.0 equiv. pyridine

3 wt \% PS-750-M in $\mathrm{H}_{2} \mathrm{O}$

$(0.5 \mathrm{M}), 60^{\circ} \mathrm{C}, 35 \mathrm{~min}$

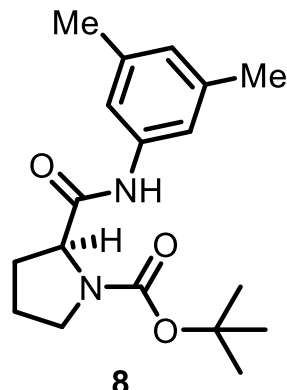

$91 \%, 13.4 \mathrm{~g}$ (average of two runs)

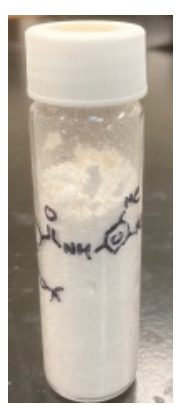

8 (isolated)

$5.6 \mathrm{~g}(46.5 \mathrm{mmol}, 1.0$ equiv. $)$

Scheme S2. Multi-gram synthesis of intermediate 8 for ACT-462206.

(tert-Butoxycarbonyl)-L-proline (7) (10 g, $46.5 \mathrm{mmol}, 1$ equiv.), 3,5-dimethylaniline (4) (5.63 g, $46.5 \mathrm{mmol}, 1$ equiv.) and $\mathrm{EDC} \cdot \mathrm{HCl}(11.6 \mathrm{~g}, 60.45 \mathrm{mmol}, 1.3$ equiv.) were taken in an oven-dried $250 \mathrm{~mL}$ round-bottom flask equipped with a PTFE-coated magnetic stir bar. Reaction flask was closed with a rubber septum and $90 \mathrm{~mL} 3 \mathrm{wt} \%$ aqueous PS-750-M was added to the reaction mixture. Septum was wrapped with PTFE tape and a parafilm. Pyridine $(7.5 \mathrm{~mL}, 93 \mathrm{mmol}, 2$ equiv.) was subsequently added to the reaction mixture and mixture was stirred at $60{ }^{\circ} \mathrm{C}$ on preheated oil bath for 35 minutes. A color change from red to pale yellow was observed. After reaction completion as monitored by TLC (ethyl acetate/hexane, 2:3), stirring was stopped and reaction mixture was cooled to rt. The reaction mixture was extracted with 3 x $30 \mathrm{~mL}$ EtOAc and combined organic layers were dried over anhydrous $\mathrm{Na}_{2} \mathrm{SO}_{4}$. The solvent was removed under high vacuum. Crude product was purified by flash chromatography over silica gel using hexanes/EtOAc as eluent (1:1 EtOAc:hexane) to obtain tert-butyl 2-((3,5-dimethylphenyl)carbamoyl) pyrrolidine-1carboxylate (8) as white solid, yield $13.40 \mathrm{~g}$ (91\%, average of two runs), $\mathrm{R}_{f} 0.4$ (2:3, hexanes/ethyl acetate). ${ }^{1}$ H NMR (400 MHz, DMSO-d6) $\delta 9.78$ (s, 1H), 7.23 (s, 2H), 6.68 (s, 1H), 4.34 - 4.09 $(\mathrm{m}, 1 \mathrm{H}), 3.52-3.38(\mathrm{~m}, 1 \mathrm{H}), 3.36-3.23(\mathrm{~m}, 1 \mathrm{H}), 2.22(\mathrm{~s}, 6 \mathrm{H}), 2.19-2.11(\mathrm{~m}, 1 \mathrm{H}), 1.95-1.69$ (m, 3H), 1.40 (s, 3H), 1.28 (s, 6H). ${ }^{13}$ C NMR (100 MHz, DMSO-d6) Rotomers: $\delta$ 171.3, 170.9, 153.6, 153.1, 138.9, 137.5, 124.7, 124.6, 117, 116.9, 78.6, 78.4, 60.3, 60, 46.7, 46.5, 31, 30.2, 28.1, 27.9, 23.9, 23.3, 21.1. ${ }^{5,6}$ Product purity $(98.20 \%)$ was determined by HPLC with CHIRALCEL $^{\circledR}$ 
AD-H column (hexane $/ i$-PrOH $=90 / 10$ ), flow rate $=1 \mathrm{~mL} / \mathrm{min}, 25{ }^{\circ} \mathrm{C}$. Process mass intensity $(\mathrm{PMI})=26.74(\text { calculated according to literature procedure })^{4}$ 

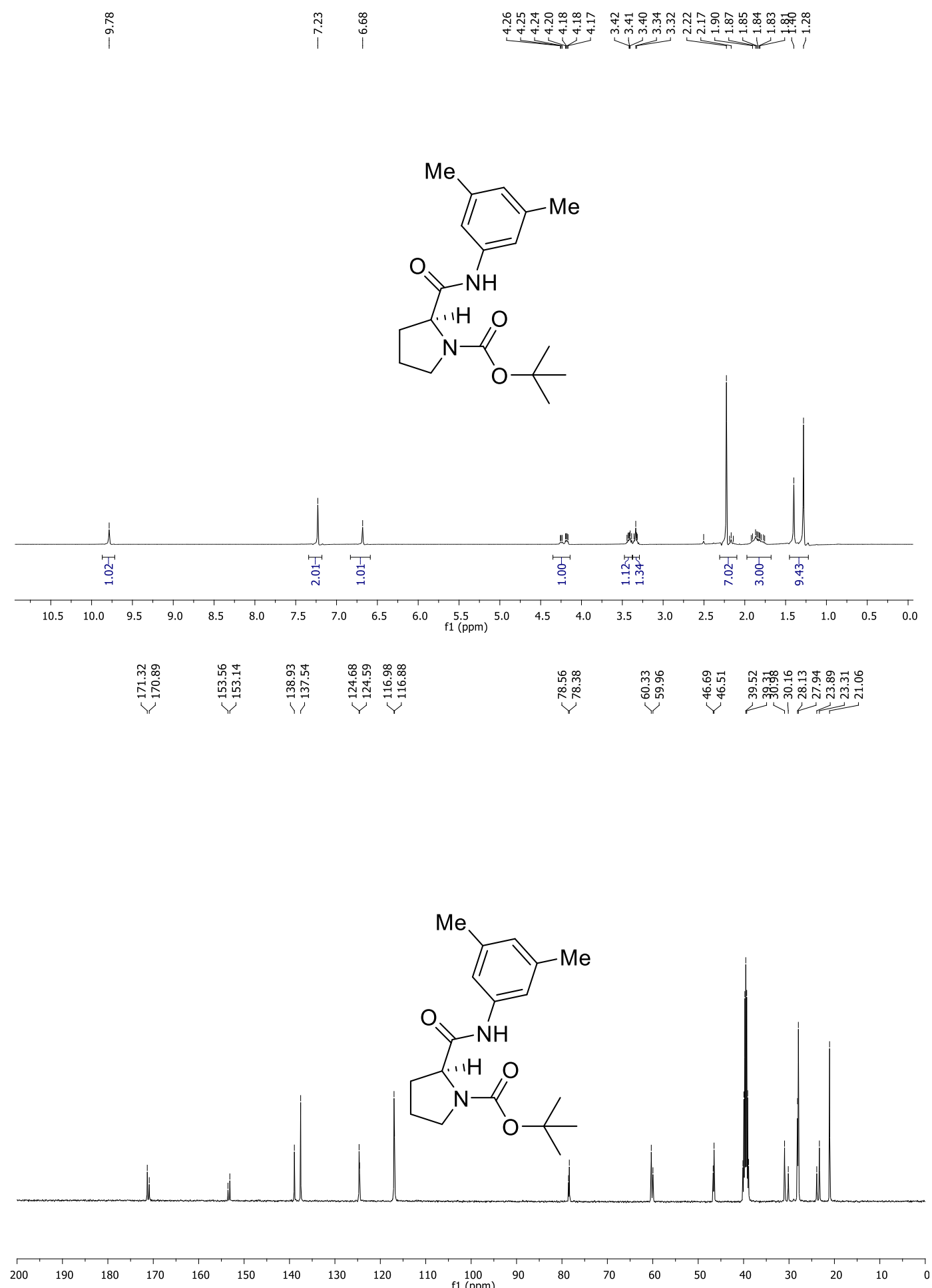

Figure S6. ${ }^{1} \mathrm{H}$ and ${ }^{13} \mathrm{C}$ NMR of 8 . 


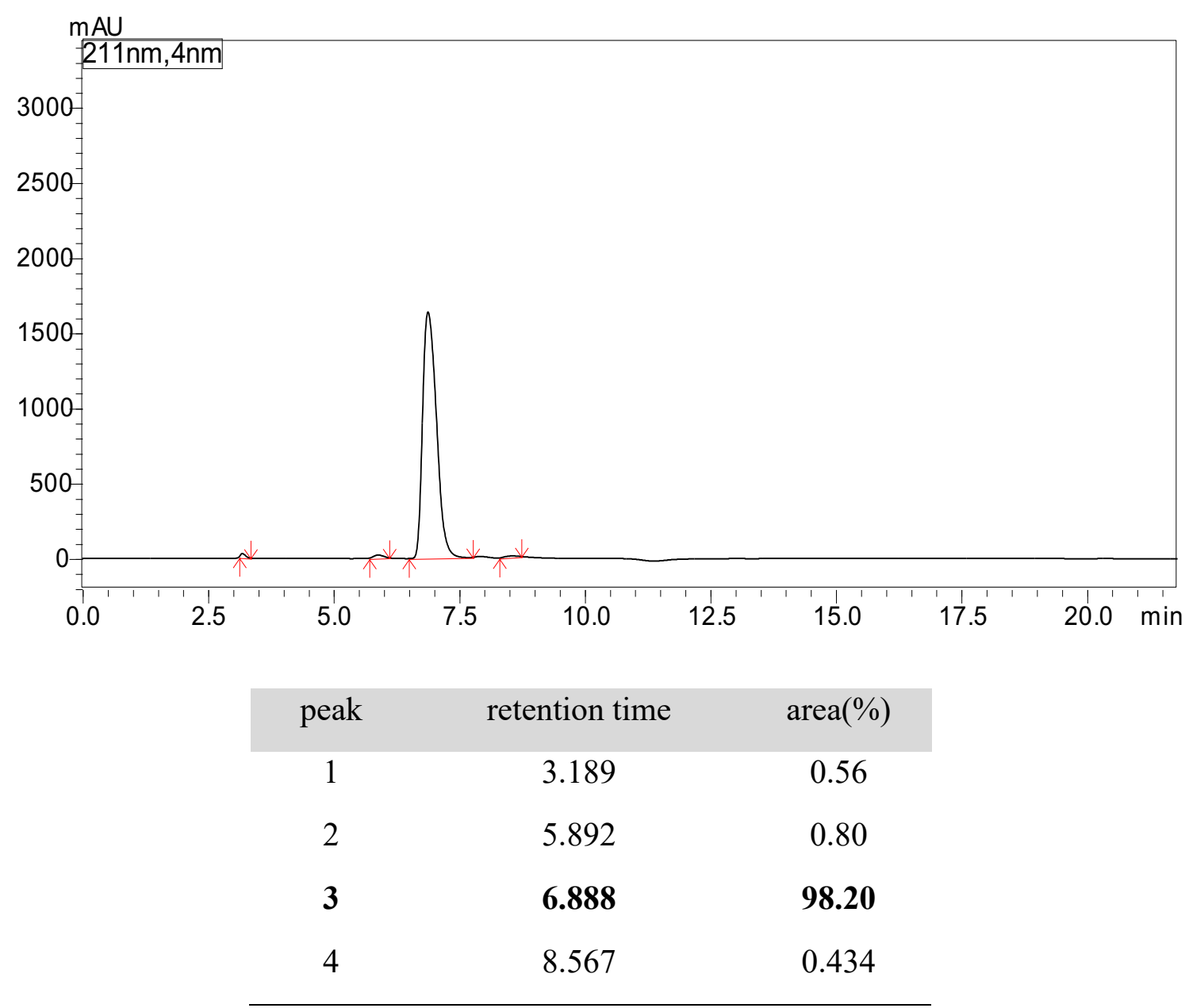

Figure S7. Purity analysis of 8 by HPLC. 


\section{MULTI-GRAM SYNTHESIS OF 3}
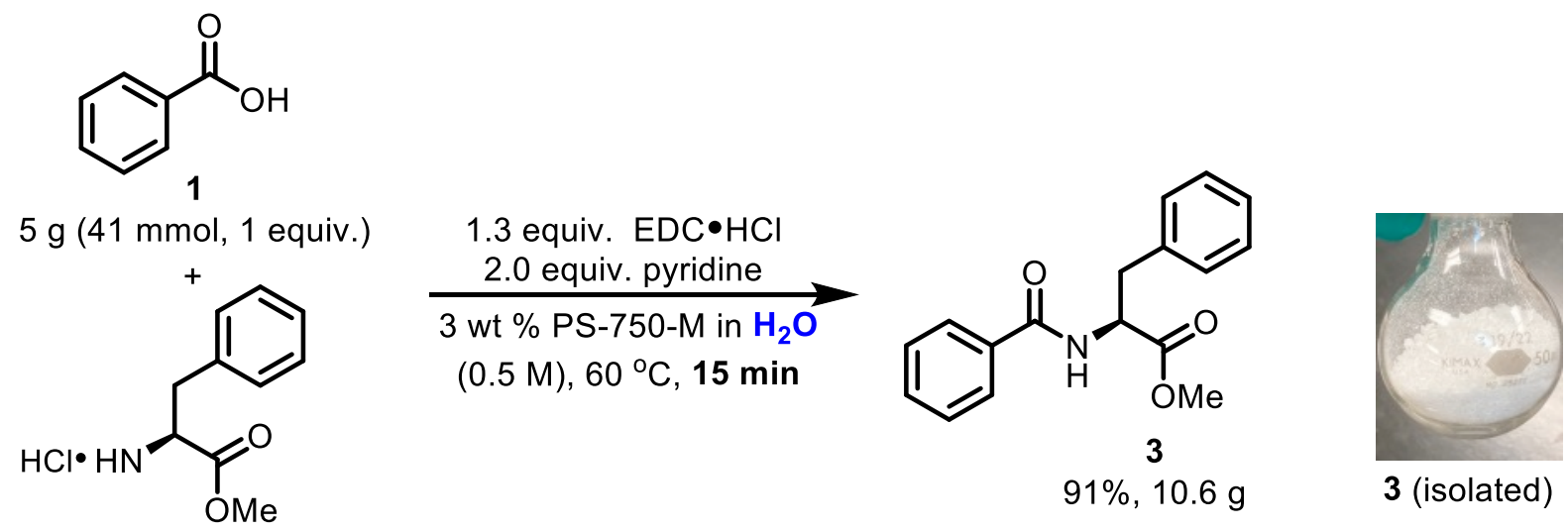

2

$8.8 \mathrm{~g}(41 \mathrm{mmol}, 1.0$ equiv. $)$

Scheme S3. Multi-gram synthesis of 3.

Benzoic acid (1) (5 g, $41 \mathrm{mmol}, 1$ equiv.), L-phenylalaninate hydrochloride (8.8 g, $41 \mathrm{mmol}, 1$ equiv.) and $\mathrm{EDC} \cdot \mathrm{HCl}(10.22 \mathrm{~g}, 53.3 \mathrm{mmol}, 1.3$ equiv.) were taken in an oven-dried $250 \mathrm{~mL}$ roundbottom flask equipped with a PTFE-coated magnetic stir bar. Reaction flask was closed with a rubber septum and $82 \mathrm{~mL} 3 \mathrm{wt} \%$ aqueous PS-750-M was added to the reaction mixture. Septum was wrapped with PTFE tape and a parafilm. Pyridine $(6.6 \mathrm{~mL}, 82 \mathrm{mmol}, 2$ equiv.) was subsequently added to the reaction mixture and mixture was stirred at $60^{\circ} \mathrm{C}$ on pre-heated oil bath for 15 minutes. After reaction completion as monitored by TLC (ethyl acetate/hexane, 1:1), stirring was stopped and reaction mixture was cooled to rt. Solid was filtered through a Whatman filter paper and washed with deionized water $(3 \times 30 \mathrm{~mL})$. Collected solid was dried under reduced pressure to obtain methyl benzoyl- $L$-phenylalaninate (3) as final product. White solid, yield 10.6 g (91\%), $\mathrm{R}_{f} 0.4$ (1:1, hexanes/ethyl acetate). ${ }^{1} \mathbf{H}$ NMR (400 MHz, $\left.\mathbf{C D C l}_{3}\right) \delta 7.69(\mathrm{~d}, J=7.2 \mathrm{~Hz}$, 2H), 7.48 (t, $J=7.4 \mathrm{~Hz}, 1 \mathrm{H}), 7.39$ (t, $J=7.5 \mathrm{~Hz}, 2 \mathrm{H}), 7.32-7.16(\mathrm{~m}, 3 \mathrm{H}), 7.10(\mathrm{~d}, J=6.5 \mathrm{~Hz}$, 2H), $6.54(\mathrm{~d}, J=7.5 \mathrm{~Hz}, 1 \mathrm{H}), 5.25-4.93(\mathrm{~m}, 1 \mathrm{H}), 3.74(\mathrm{~s}, 3 \mathrm{H}), 3.23(\mathrm{~m}, 2 \mathrm{H}) .{ }^{13} \mathbf{C}$ NMR (100 MHz, $\left.\mathbf{C D C l}_{3}\right) \delta 172.2,166.7,140.7,134.4,131.8,128.9,128.7,127.8,127.2,126.4,52.0,49.9$, 39.8. ${ }^{3}$ Product purity $(97.7 \%)$ was determined by HPLC with CHIRALCEL ${ }^{\circledR}$ OD-H column (hexane $/ i-\mathrm{PrOH}=90 / 10$ ), flow rate $=1 \mathrm{~mL} / \mathrm{min}, 40{ }^{\circ} \mathrm{C} . \mathrm{PMI}=19.36$ (calculated according to the literature procedure $)^{3}$. 

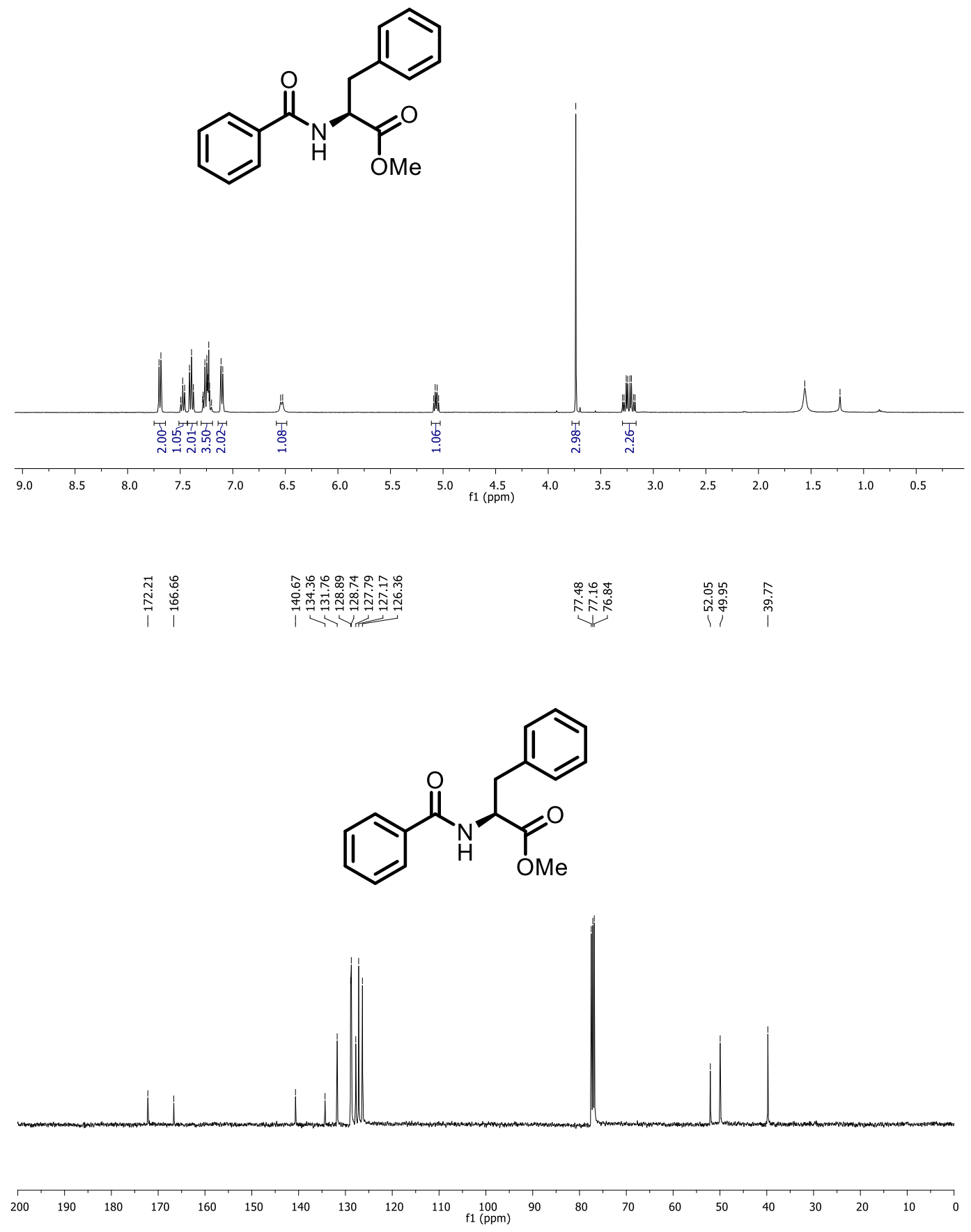

Figure S8. ${ }^{1} \mathrm{H}$ and ${ }^{13} \mathrm{C}$ NMR of 3 . 


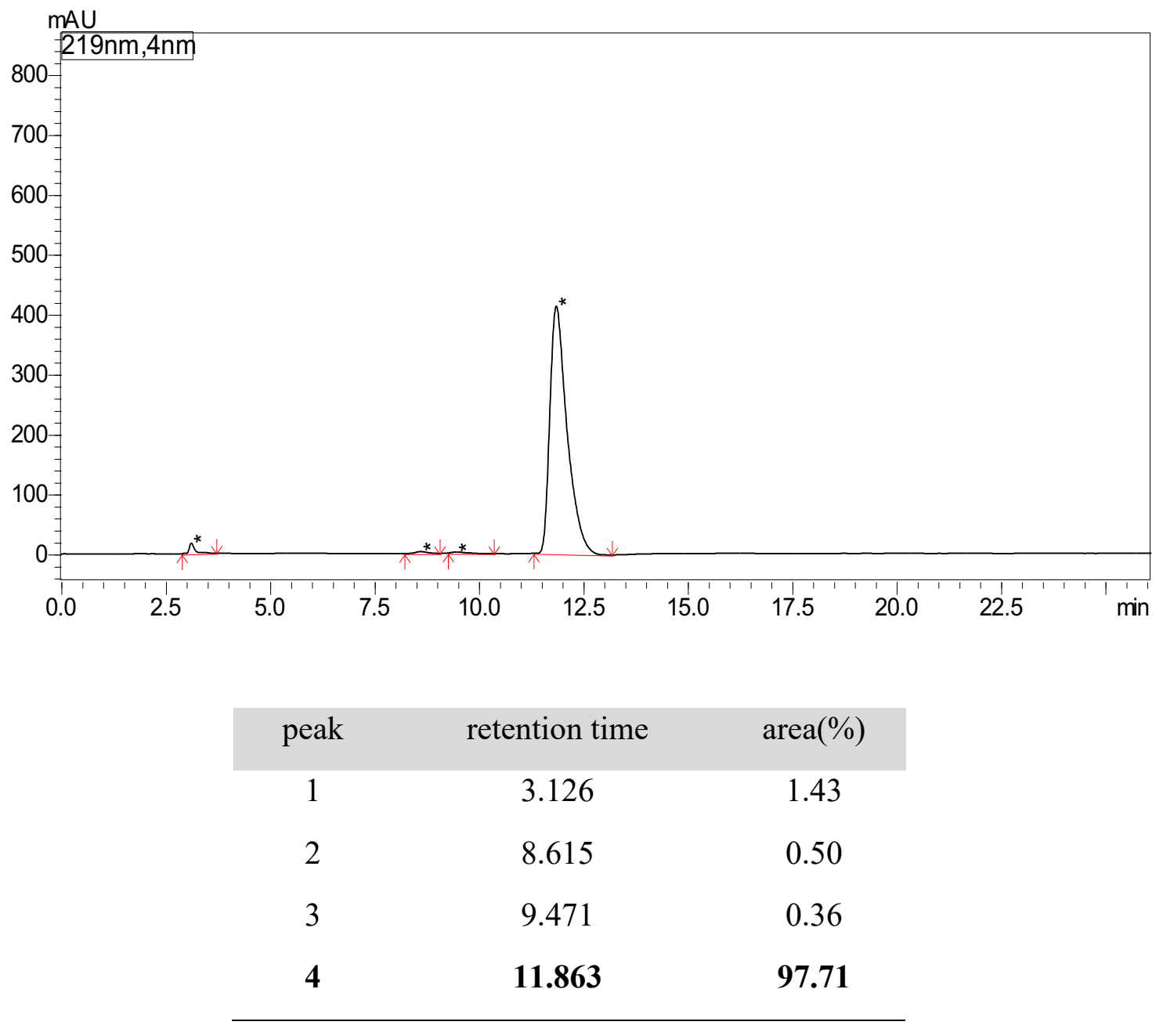

Figure S9. Purity analysis of $\mathbf{3}$ by HPLC. 


\section{MULTI-GRAM SYNTHESIS OF 11}<smiles>CC(C)C(NC(=O)OCc1ccccc1)C(=O)O</smiles>

$7 \mathrm{~g}(27.8 \mathrm{mmol}, 1.0$ equiv. $)$<smiles>[13CH3]</smiles><smiles>Nc1ccccc1</smiles>

10
1.3 equiv. $\mathrm{EDC} \bullet \mathrm{HCl}$

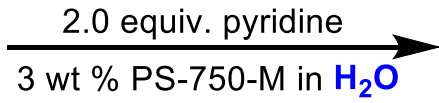

$(0.5 \mathrm{M}), 60^{\circ} \mathrm{C}, 25 \mathrm{~min}$

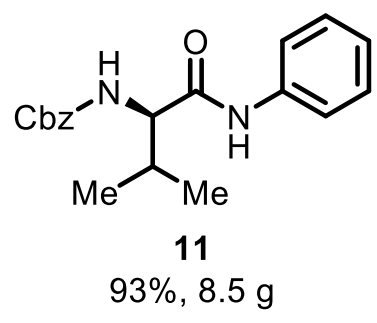

$93 \%, 8.5 \mathrm{~g}$

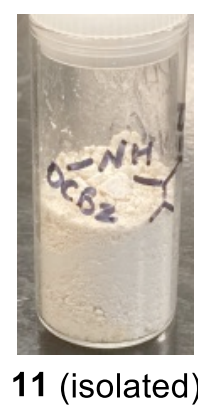

11 (isolated)

$2.6 \mathrm{~g}(27.8 \mathrm{mmol}, 1.0$ equiv. $)$

Scheme S4. Multi-gram synthesis of 11.

((Benzyloxy)carbonyl)- $D$-valine (1) (7 g, 27.8 mmol, 1 equiv.), aniline (2.6 g, 27.8 mol, 1 equiv.) and $\mathrm{EDC} \cdot \mathrm{HCl}$ (6.9 g, $36.18 \mathrm{mmol}, 1.3$ equiv.) were taken in an oven-dried $250 \mathrm{~mL}$ round-bottom flask equipped with a PTFE-coated magnetic stir bar. Reaction flask was closed with a rubber septum and $56 \mathrm{~mL} 3 \mathrm{wt} \%$ aqueous PS-750-M was added to the reaction mixture. Septum was wrapped with PTFE tape and a parafilm. Pyridine (4.5 mL, $55.7 \mathrm{mmol}, 2$ equiv.) was subsequently added to the reaction mixture and mixture was stirred at $60{ }^{\circ} \mathrm{C}$ on pre-heated oil bath for 25 minutes. After reaction completion as monitored by TLC (ethyl acetate/hexane, 1:1), stirring was stopped and reaction mixture was cooled to rt. Solid was filtered through a Whatman filter paper and washed with deionized water $(3 \times 40 \mathrm{~mL})$. Collected solid was dried under reduced pressure to obtain benzyl (D)-(3-methyl-1-oxo-1-(phenylamino)butan-2-yl)carbamate (11) as final product. Off-white solid, yield $8.5 \mathrm{~g}(93 \%), \mathrm{R}_{f} 0.4$ (1:1, hexanes/ethyl acetate). ${ }^{1} \mathrm{H}$ NMR (400 MHz, $\left.\mathbf{C D C l}_{3}\right) \delta 7.87(\mathrm{~s}, 1 \mathrm{H}), 7.41(\mathrm{~d}, J=7.8 \mathrm{~Hz}, 2 \mathrm{H}), 7.33-7.12(\mathrm{~m}, 7 \mathrm{H}), 7.03(\mathrm{t}, J=7.4 \mathrm{~Hz}, 1 \mathrm{H}), 5.38$ (s, 1H), $5.12-4.93(\mathrm{~m}, 2 \mathrm{H}), 4.02(\mathrm{t}, J=7.4 \mathrm{~Hz}, 1 \mathrm{H}), 2.29-2.00(\mathrm{~m}, 1 \mathrm{H}), 0.96(\mathrm{~d}, J=6.7 \mathrm{~Hz}$, 3H), $0.92(\mathrm{~d}, J=6.8 \mathrm{~Hz}, 3 \mathrm{H}) .{ }^{13} \mathbf{C}$ NMR (100 MHz, $\left.\mathbf{C D C l}_{3}\right) \delta 169.9,156.9,137.5,136.2,129.1$, 128.7, 128.4, 128.2, 124.7, 120.3, 67.5, 61.6, 31.0, 19.5, 18.2. ${ }^{3}$ Product purity $(98.16 \%)$ was determined by HPLC with CHIRALCEL ${ }^{\circledR}$ OJ-H column (hexane $\left./ i-\mathrm{PrOH}=90 / 10\right)$, flow rate $=1$ $\mathrm{mL} / \mathrm{min}, 40^{\circ} \mathrm{C} . \mathrm{PMI}=19.83$ (was calculated according to the literature procedure) ${ }^{4}$ 

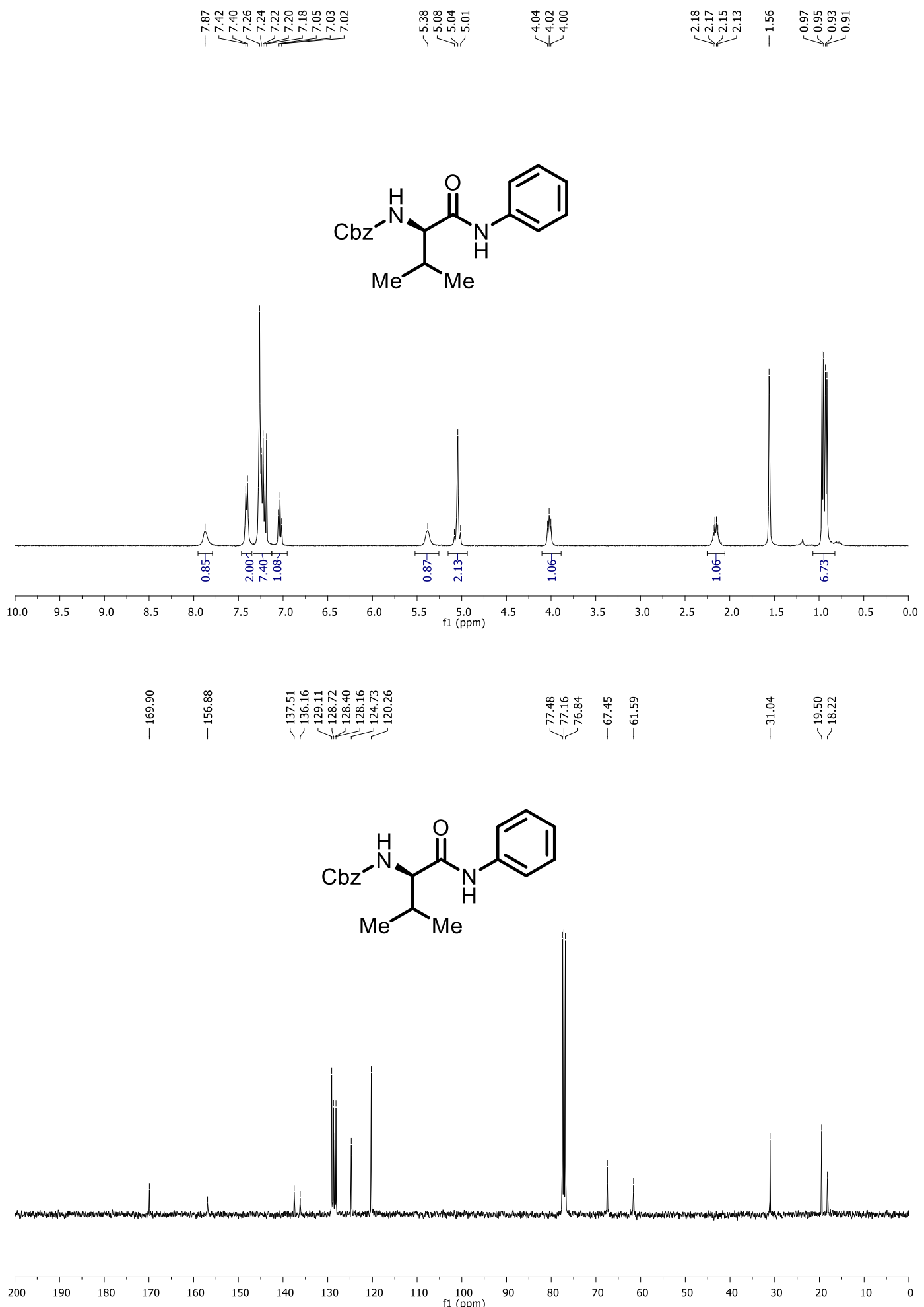

Figure S10. ${ }^{1} \mathrm{H}$ and ${ }^{13} \mathrm{C}$ NMR of 11. 


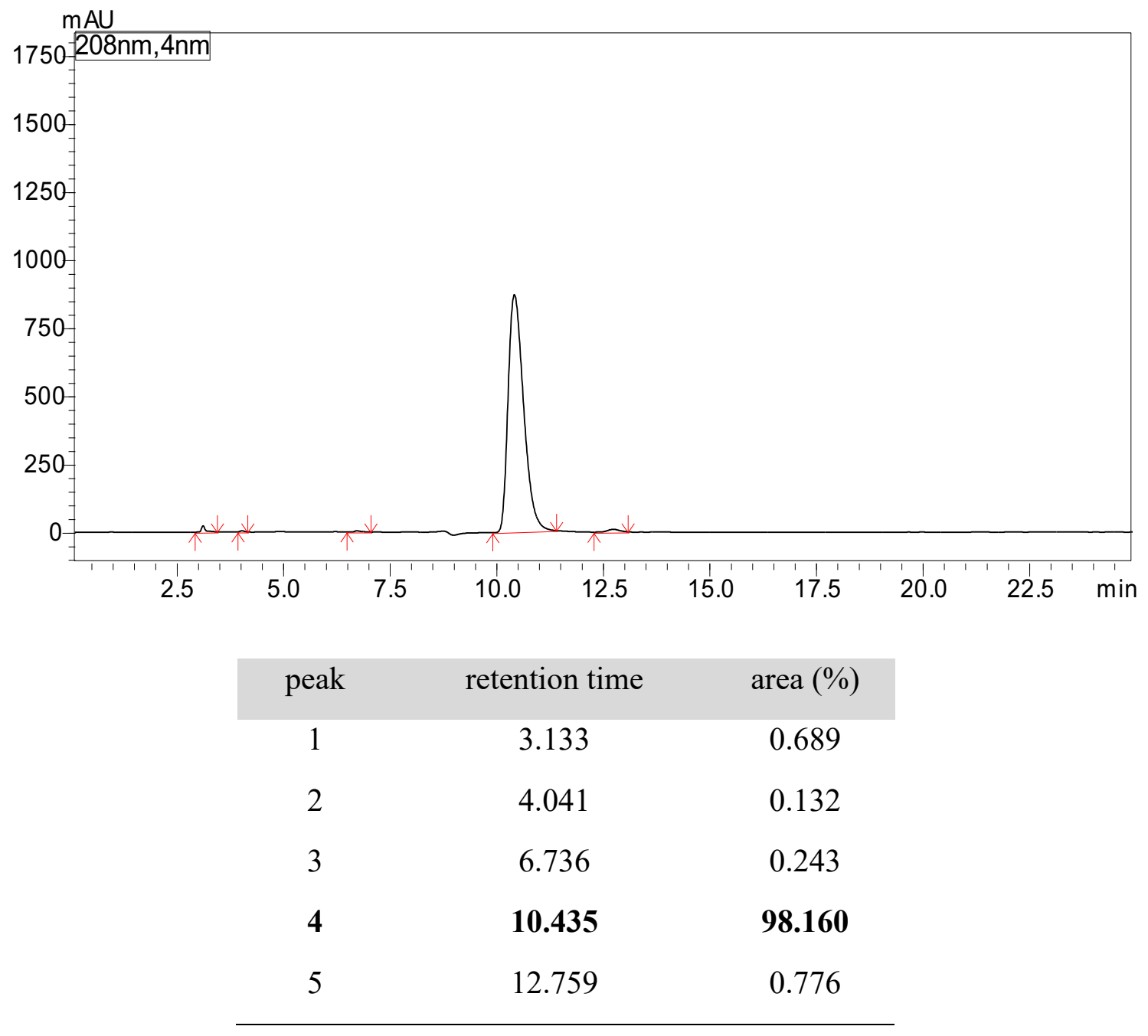

Figure S11. Purity analysis of 11 by HPLC. 


\section{EPIMERIZATION STUDY ON GRAM-SCALE}

\section{Methyl benzoyl-DL-phenylalaninate standard (rac-3)}

Enantiomeric ratio was determined by HPLC with CHIRALCEL ${ }^{\circledR}$ OD-H column (hexane/i$\operatorname{PrOH}=90 / 10)$, flow rate $=1 \mathrm{~mL} / \mathrm{min}, 40^{\circ} \mathrm{C}$.
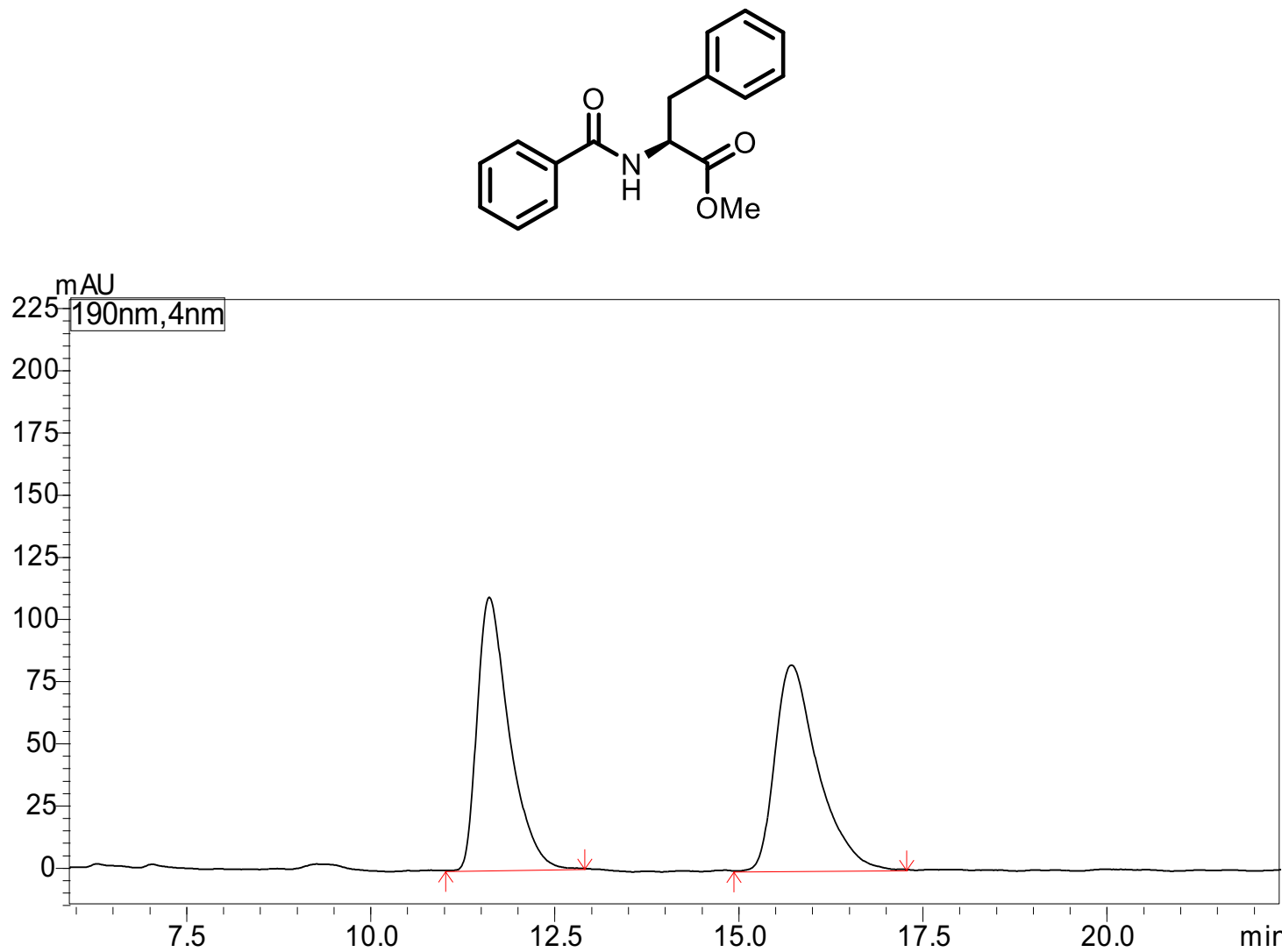

\begin{tabular}{|c|c|c|c|}
\hline peak & product & retention time & $\operatorname{area}(\%)$ \\
\hline 1 & $\begin{array}{c}\text { Methyl benzoyl- } L \text {-phenylalaninate } \\
\text { standard }\end{array}$ & 11.625 & 49.838 \\
\hline 2 & $\begin{array}{c}\text { Methyl benzoyl- } D \text {-phenylalaninate } \\
\text { standard }\end{array}$ & 15.73 & 50.162 \\
\hline
\end{tabular}

Figure S12. HPLC analysis of rac-3. 
Methyl benzoyl- $L$-phenylalaninate standard (3)

Enantiomeric ratio was determined by HPLC with CHIRALCEL ${ }^{\circledR}$ OD-H column (hexane/i$\mathrm{PrOH}=90 / 10)$, flow rate $=1 \mathrm{~mL} / \mathrm{min}, 40{ }^{\circ} \mathrm{C}$

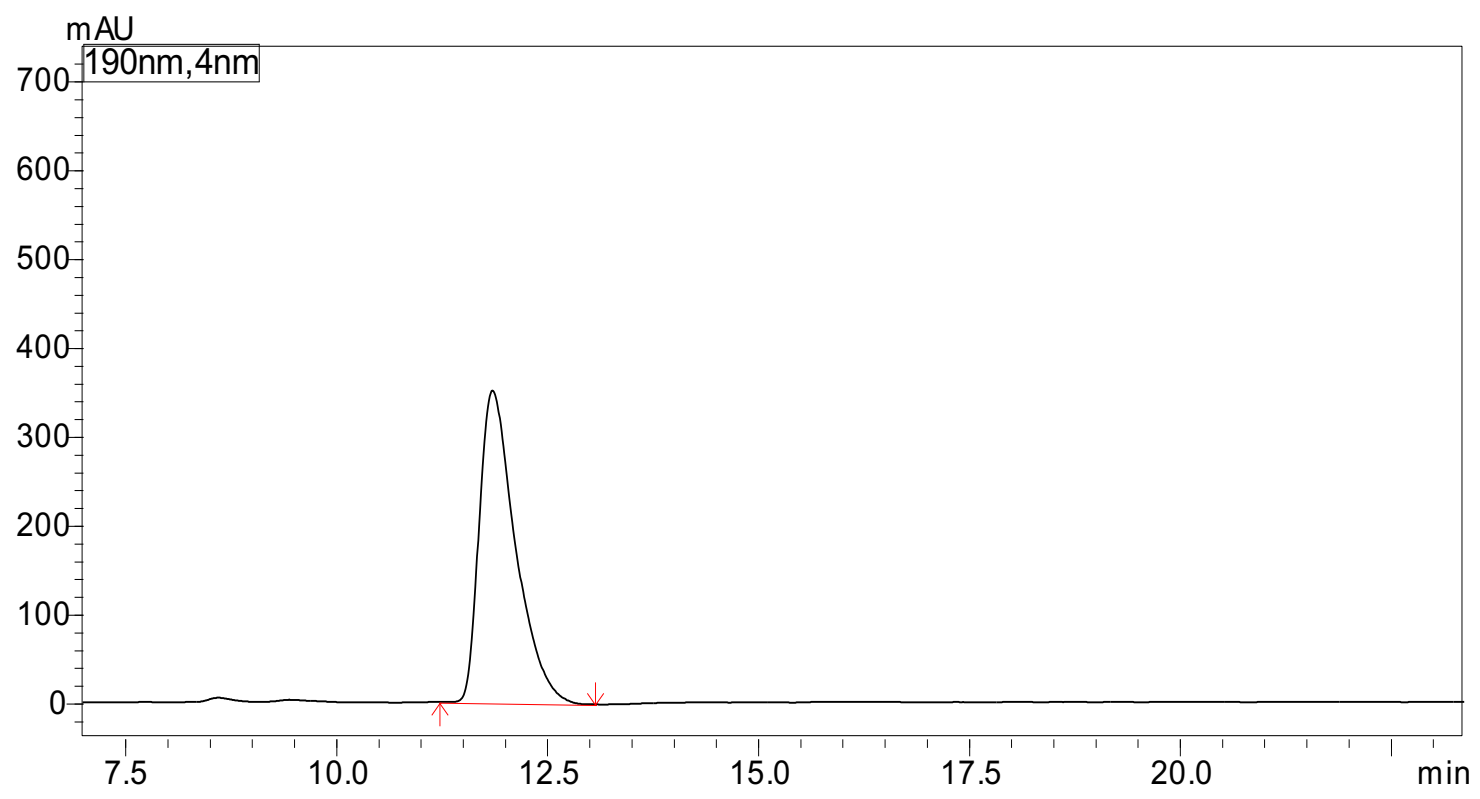

\begin{tabular}{cccc} 
peak & product & retention time & area(\%) \\
1 & Methyl benzoyl- $L$-phenylalaninate & 11.863 & 100 \\
standard & & \\
\hline
\end{tabular}

Figure S13. HPLC analysis of 3 


\section{Benzyl (DL)-(3-methyl-1-oxo-1-(phenylamino)butan-2-yl)carbamate ( $r a c-11)$}

Enantiomeric ratio was determined by HPLC with CHIRALCEL ${ }^{\circledR} \mathrm{OJ}-\mathrm{H}$ column (hexane $/ i-\mathrm{PrOH}=$ 90/10), flow rate $=1 \mathrm{~mL} / \mathrm{min}, 40^{\circ} \mathrm{C}$<smiles>CC(C)[C@H](NC(=O)OCc1ccccc1)C(=O)Nc1ccccc1</smiles>

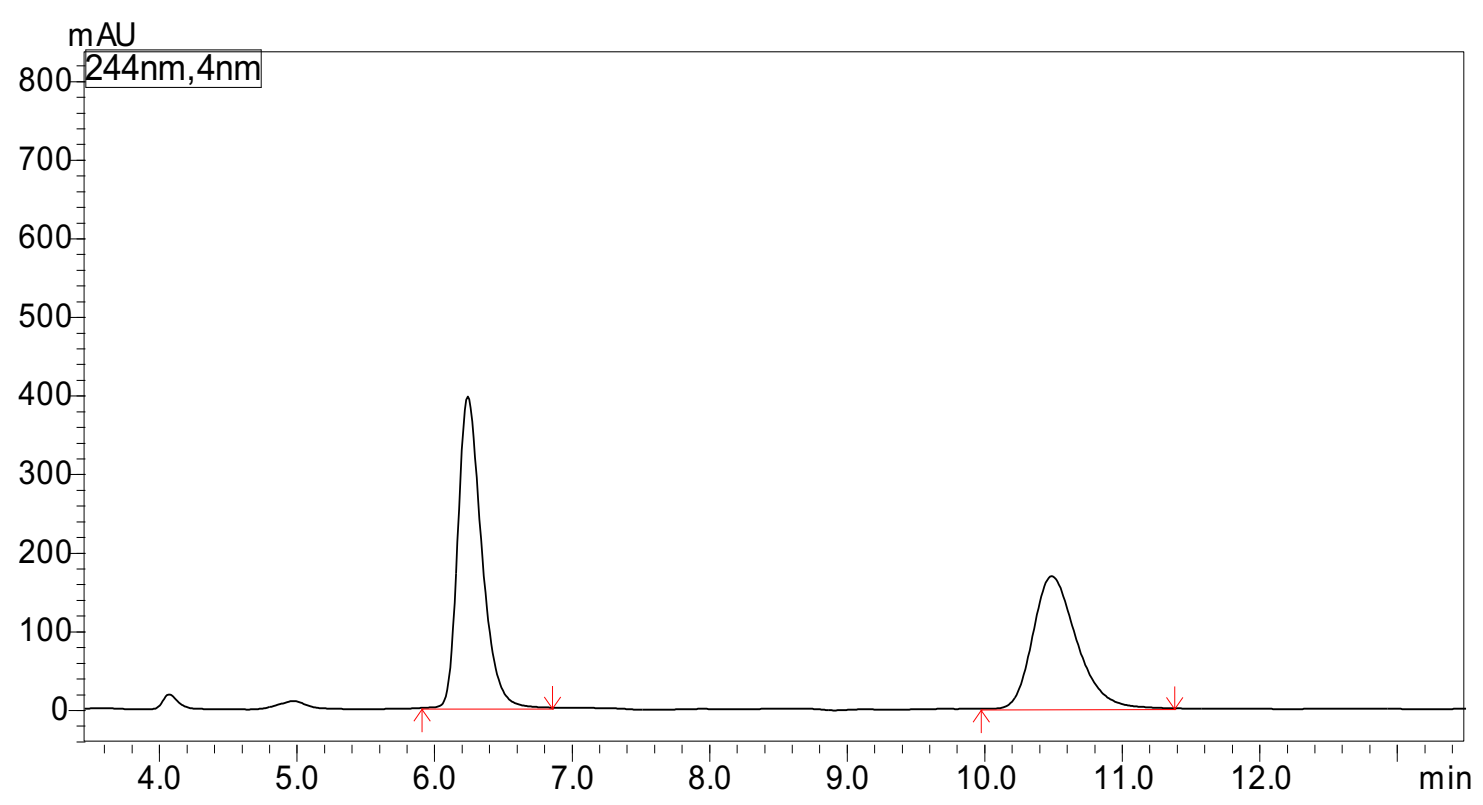

\begin{tabular}{cccc} 
peak & product & retention time & area(\%) \\
1 & $\begin{array}{c}\text { Benzyl }(L)-(3-\text { methyl-1-oxo-1- } \\
\text { (phenylamino)butan-2-yl)carbamate }\end{array}$ & 6.251 & 49.31 \\
2 & $\begin{array}{c}\text { Benzyl }(D)-(3-\text { methyl-1-oxo-1- } \\
\text { (phenylamino)butan-2-yl)carbamate }\end{array}$ & 10.497 & 50.69 \\
\hline
\end{tabular}

Figure S14. HPLC analysis of $r a c-11$. 


\section{Benzyl (DL)-(3-methyl-1-oxo-1-(phenylamino)butan-2-yl)carbamate (11)}

Enantiomeric ratio was determined by HPLC with CHIRALCEL ${ }^{\circledR}$ OJ-H column (hexane $/ i$-PrOH= 90/10), flow rate $=1 \mathrm{~mL} / \mathrm{min}, 40^{\circ} \mathrm{C}$

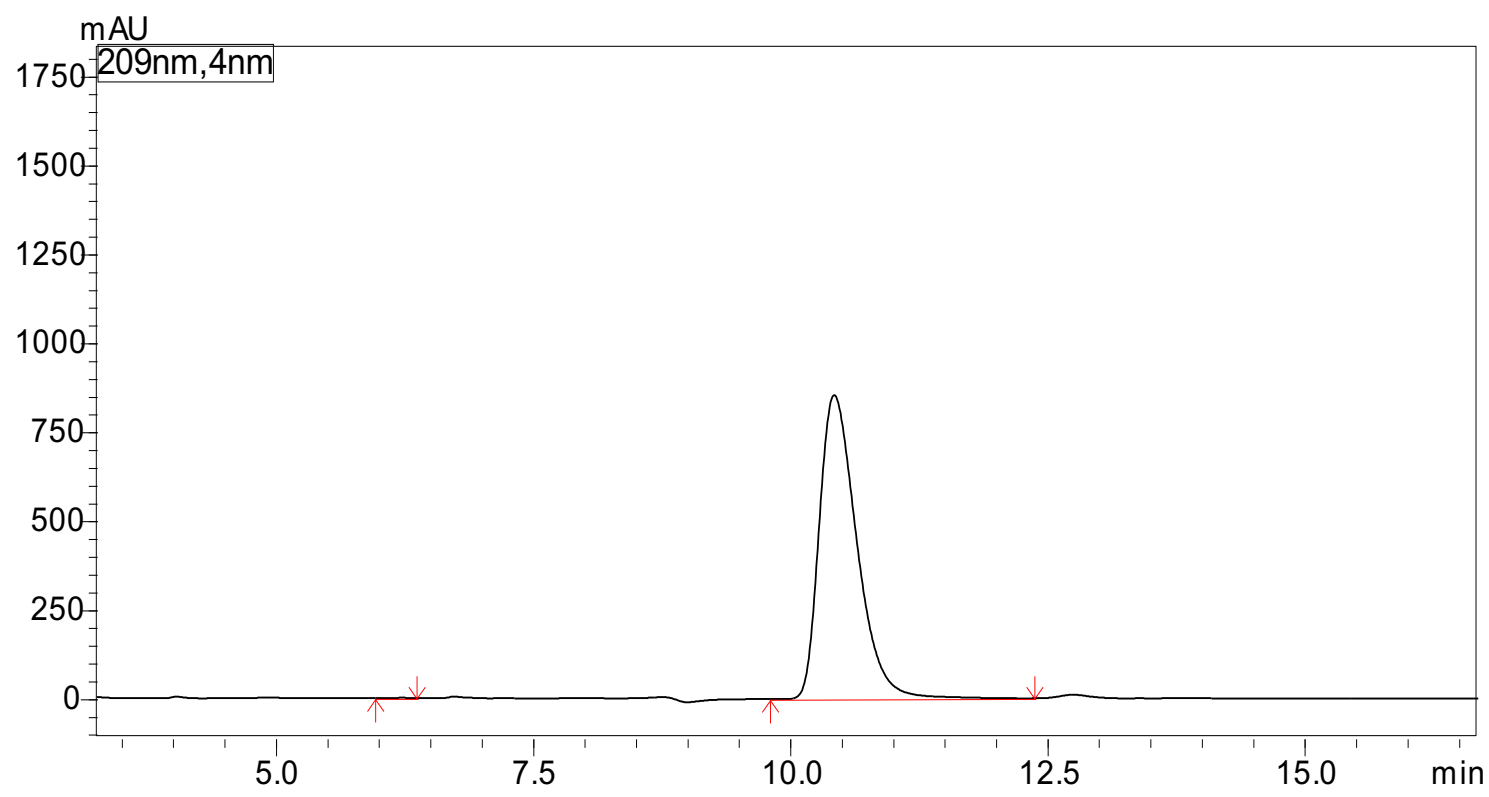

\begin{tabular}{cccc} 
peak & product & retention time & area $(\%)$ \\
1 & $\begin{array}{c}\text { Benzyl }(L)-(3-\text {-methyl-1-oxo-1- } \\
\text { (phenylamino)butan-2-yl)carbamate }\end{array}$ & 6.234 & 0.047 \\
1 & $\begin{array}{c}\text { Benzyl }(D)-(3-m e t h y l-1-\text { oxo-1- } \\
(\text { phenylamino)butan-2-yl)carbamate }\end{array}$ & 10.435 & 99.95 \\
\hline
\end{tabular}

Figure S15. HPLC analysis of 11. 


\section{NMR EXPERIMENTS}

\section{a) NMR study in $\mathrm{CDCl}_{3}$ as $\mathrm{NMR}$ solvent}

Sample preparation. All samples were stirred at room temperature for 15 minutes. All experiments were carried out in Varian Innova $400 \mathrm{MHz} \mathrm{NMR}$ at $25{ }^{\circ} \mathrm{C}$ using $\mathrm{CDCl}_{3}$ as $\mathrm{NMR}$ solvent.

Table S1. Sample details for ${ }^{1} \mathrm{H}$ NMR experiments

\begin{tabular}{|c|c|}
\hline Sample ID & constituents \\
\hline Sample A & $\mathrm{EDC} \cdot \mathrm{HCl}$ \\
\hline Sample B & 1 equiv $\mathrm{EDC} \cdot \mathrm{HCl}+1$ equiv $\mathrm{Et}_{3} \mathrm{~N}$ \\
\hline Sample C & 1 equiv $\mathrm{EDC} \cdot \mathrm{HCl}+1$ equiv $i$-Pr $2 \mathrm{NEt}$ \\
\hline Sample D & 1 equiv $\mathrm{EDC} \cdot \mathrm{HCl}+1$ equiv 2,6 -lutidine \\
\hline Sample E & 1 equiv $\mathrm{EDC} \cdot \mathrm{HCl}+1$ equiv collidine \\
\hline Sample $\mathrm{F}$ & 1 equiv $\mathrm{EDC} \cdot \mathrm{HCl}+1$ equiv pyridine \\
\hline
\end{tabular}




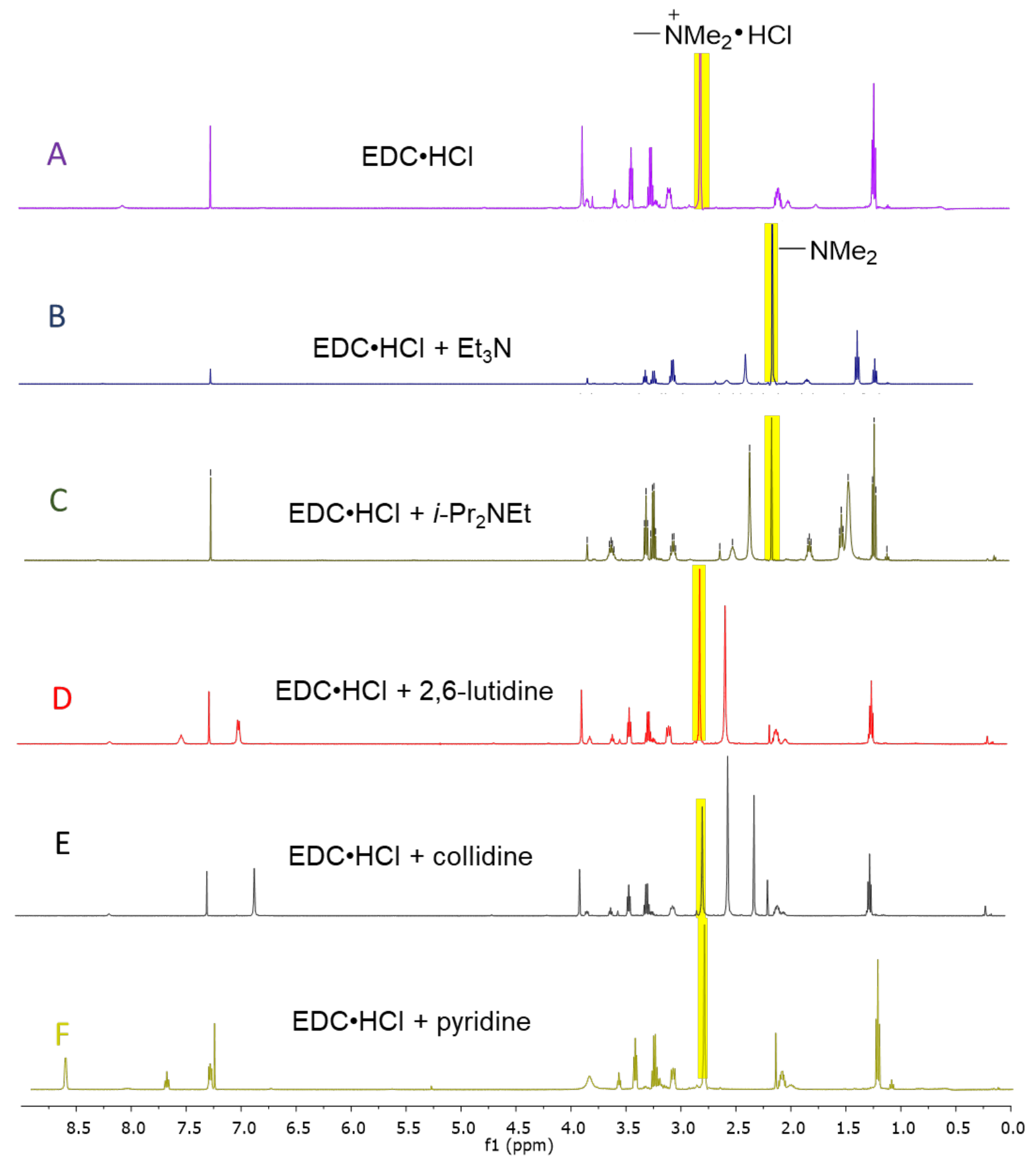

Figure S16. Effect of bases on EDC $\cdot \mathrm{HCl}$ structure. (A) EDC $\cdot \mathrm{HCl}$. (B) EDC $\cdot \mathrm{HCl}$ and $\mathrm{Et}_{3} \mathrm{~N}$. (C) $\mathrm{EDC} \cdot \mathrm{HCl}$ and $i$ - $\mathrm{Pr}_{2} \mathrm{NEt}$ (D) $\mathrm{EDC} \cdot \mathrm{HCl}$ and 2,6-lutidine. (E) $\mathrm{EDC} \cdot \mathrm{HCl}$ and collidine (F) $\mathrm{EDC} \cdot \mathrm{HCl}$ and pyridine. 


\section{b) NMR study in $\mathrm{D}_{2} \mathrm{O}$ as $\mathrm{NMR}$ solvent}

Sample preparation. All samples were stirred at room temperature for 15 minutes. All experiments were carried out in Varian Innova $400 \mathrm{MHz} N M R$ at $25{ }^{\circ} \mathrm{C}$ using $\mathrm{D}_{2} \mathrm{O}$ as $\mathrm{NMR}$ solvent.

Table S2. Sample details for ${ }^{1} \mathrm{H}$ NMR experiment

\begin{tabular}{|c|c|}
\hline Sample ID & constituents \\
\hline Sample A & $\mathrm{EDC} \cdot \mathrm{HCl}$ \\
\hline Sample B & 1 equiv $\mathrm{EDC} \cdot \mathrm{HCl}+1$ equiv $\mathrm{Et}_{3} \mathrm{~N}$ \\
\hline Sample C & 1 equiv $\mathrm{EDC} \cdot \mathrm{HCl}+1$ equiv $i$-Pr $2 \mathrm{NEt}$ \\
\hline Sample D & 1 equiv $\mathrm{EDC} \cdot \mathrm{HCl}+1$ equiv $2,6-$-lutidine \\
\hline Sample E & 1 equiv $\mathrm{EDC} \cdot \mathrm{HCl}+1$ equiv pyridine \\
\hline Sample $\mathrm{F}$ & 1 equiv $\mathrm{EDC} \cdot \mathrm{HCl}+1$ equiv collidine \\
\hline
\end{tabular}




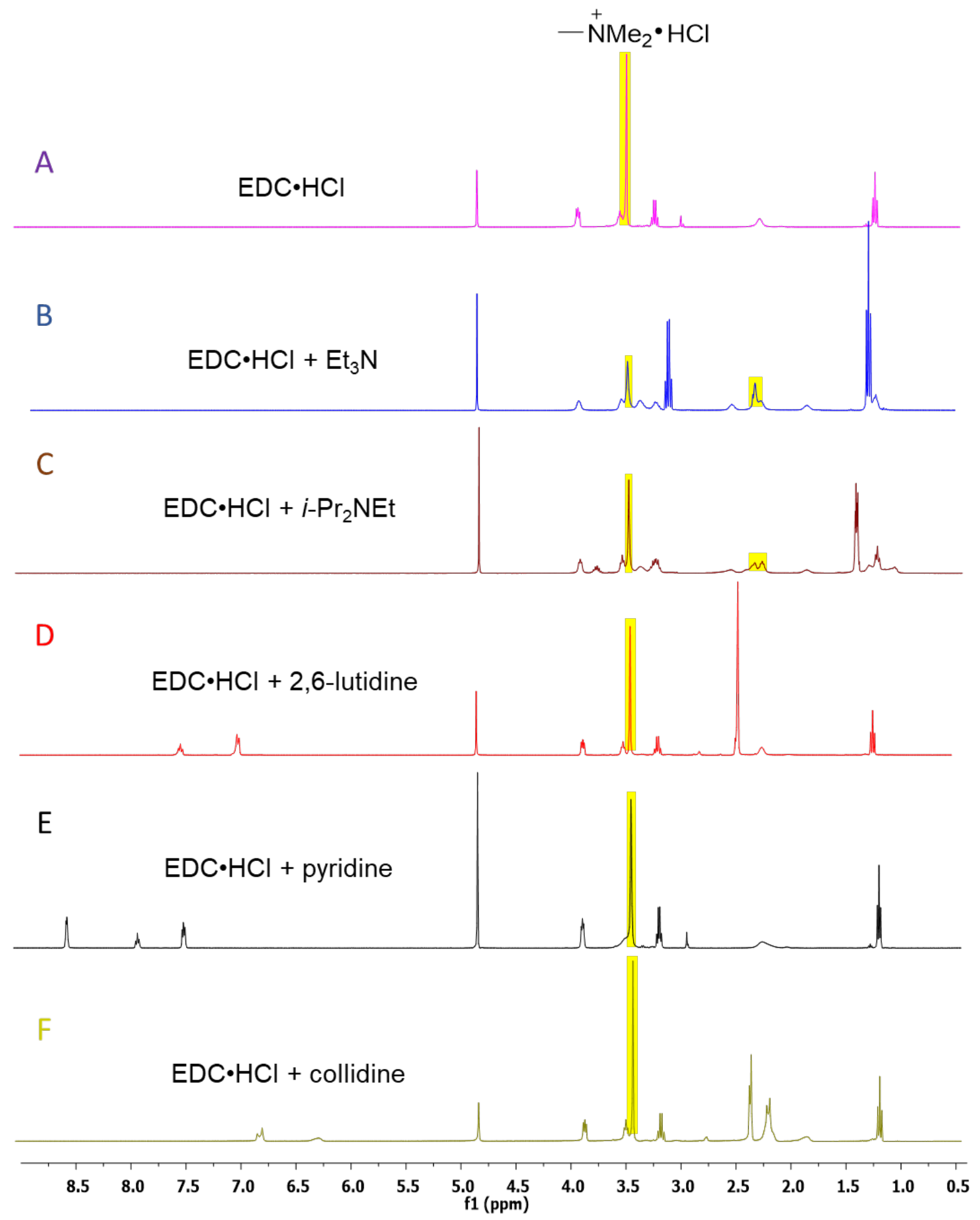

Figure S17. Effect of bases on EDC $\cdot \mathrm{HCl}$ structure. (A) EDC $\cdot \mathrm{HCl}$. (B) EDC $\cdot \mathrm{HCl}$ and $\mathrm{Et}_{3} \mathrm{~N}$. (C) $\mathrm{EDC} \cdot \mathrm{HCl}$ and $i$ - $\mathrm{Pr}_{2} \mathrm{NEt}$. (D) $\mathrm{EDC} \cdot \mathrm{HCl}$ and 2,6-lutidine. (E) $\mathrm{EDC} \cdot \mathrm{HCl}$ and pyridine (F) $\mathrm{EDC} \cdot \mathrm{HCl}$ and collidine. 


\section{CONTROL EXPERIMENT-REACTION WITHOUT BASE}

Table S3. Control experiment-reaction without base

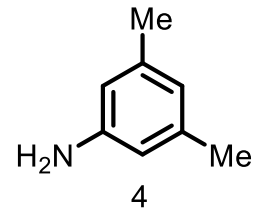

( $0.5 \mathrm{mmol}, 1.0$ equiv.)<smiles>O=C(O)Cc1ccc(O)cc1</smiles>

(0.5 mmol, 1.0 equiv)

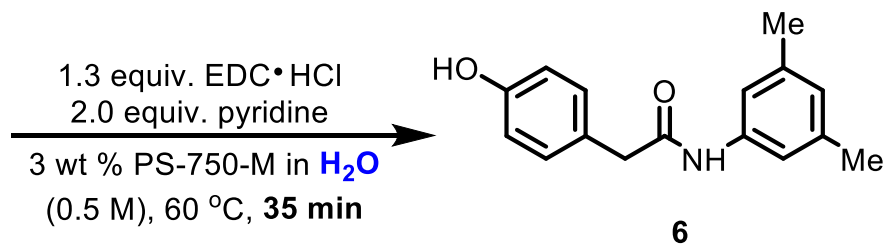

6

\begin{tabular}{ccc} 
entry & conditions & $\%$ (isolated yield) \\
1 & no deviation & 91 \\
2 & no pyridine & $39^{a}$ \\
\hline
\end{tabular}

Conditions. 4 (0.5 mmol, 1.0 equiv.), 5 (0.5 mmol, 1 equiv.), $\mathrm{EDC} \cdot \mathrm{HCl}$ (0.65 mmol, 1.3 equiv.), pyridine (1 mmol, 2.0 equiv.), (0.5 M) $1 \mathrm{~mL} 3 \mathrm{wt} \% \mathrm{PS}-750-\mathrm{M}$ in $\mathrm{H}_{2} \mathrm{O}$ at $60{ }^{\circ} \mathrm{C}, 35 \mathrm{~min}$. All yields are isolated. a) product was isolated via column chromatography using hexane:EtOAc $(1: 1)$ as eluent. 


\section{REFERENCES}

1. Brals, J.; Smith, J. D.; Ibrahim, F.; Gallou, F.; Handa, S. Micelle-Enabled Palladium Catalysis for Convenient Sp2-Sp3 Coupling of Nitroalkanes with Aryl Bromides in Water Under Mild Conditions. ACS Catal. 2017, 7, 7245-7250.

2. Fulmer, G. R.; Miller, A. J. M.; Sherden, N. H.; Gottlieb, H. E.; Nudelman, A.; Stoltz, B. M.; Bercaw, J. E.; Goldberg, K. I. NMR Chemical Shifts of Trace Impurities: Common Laboratory Solvents, Organics, and Gases in Deuterated Solvents Relevant to the Organometallic Chemist. Organometallics 2010, 29, 2176-2179.

3. Sharma, S.; Buchbinder, N. W.; Braje, W. M.; Handa, S. Fast Amide Couplings in Water: Extraction, Column Chromatography, and Crystallization Not Required. Org. Lett. 2020, $22,5737-5740$.

4. Monteith, E. R.; Mampuys, P.; Summerton, L.; Clark, J. H.; Maes, B. U. W.; McElroy, C. R. Why We Might Be Misusing Process Mass Intensity (PMI) and a Methodology to Apply It Effectively as a Discovery Level Metric. Green Chem. 2020, 22, 123-135.

5. Boss, C.; Roch-Brisbare, C.; Steiner, M. A.; Treiber, A.; Dietrich, H.; Jenck, F.; von

Raumer, M.; Sifferlen, T.; Brotschi, C.; Heidmann, B.; et al. Structure-Activity Relationship, Biological, and Pharmacological Characterization of the Proline Sulfonamide ACT-462206: A Potent, Brain-Penetrant Dual Orexin 1/Orexin 2 Receptor Antagonist. ChemMedChem 2014, 9, 2486-2496.

6. Petkova, D.; Borlinghaus, N.; Sharma, S.; Kaschel, J.; Lindner, T.; Klee, J.; Jolit, A.; Haller, V.; Heitz, S.; Britze, K.; et al. Hydrophobic Pockets of HPMC Enable Extremely Short Reaction Times in Water. ACS Sustain. Chem. Eng. 2020, 8, 12612-12617. 